\title{
Targeting the Angiopoietin/Tie Pathway: Prospects for Treatment of Retinal and Respiratory Disorders
}

\author{
Racheal Grace Akwii ${ }^{1}$ C Constantinos M. Mikelis ${ }^{1}$ (D)
}

Accepted: 6 September 2021 / Published online: 29 September 2021

(c) The Author(s), under exclusive licence to Springer Nature Switzerland AG 2021

\begin{abstract}
Anti-angiogenic approaches have significantly advanced the treatment of vascular-related pathologies. The ephemeral outcome and known side effects of the current vascular endothelial growth factor (VEGF)-based anti-angiogenic treatments have intensified research on other growth factors. The angiopoietin/Tie (Ang/Tie) family has an established role in vascular physiology and regulates angiogenesis, vascular permeability, and inflammatory responses. The Ang/Tie family consists of angiopoietins 1-4, their receptors, tie1 and 2 and the vascular endothelial-protein tyrosine phosphatase (VE-PTP). Modulation of Tie2 activation has provided a promising outcome in preclinical models and has led to clinical trials of Ang/Tie-targeting drug candidates for retinal disorders. Although less is known about the role of Ang/Tie in pulmonary disorders, several studies have revealed great potential of the Ang/Tie family members as drug targets for pulmonary vascular disorders as well. In this review, we summarize the functions of the Ang/Tie pathway in retinal and pulmonary vascular physiology and relevant disorders and highlight promising drug candidates targeting this pathway currently being or expected to be under clinical evaluation for retinal and pulmonary vascular disorders.
\end{abstract}

\section{Key Points}

The Ang/Tie signaling pathway is important for healthy and pathological retinal and respiratory vascular conditions.

Currently, there is a limited number of drug candidates targeting the Ang/Tie pathway, but with an increasing trend, due to the significance of this pathway and the need for alternative drug targets.

\section{Introduction}

The Angiopoietin-Tie (Ang/Tie) signaling pathway is a vascular-specific receptor tyrosine kinase pathway involved in vascular development. The pathway consists of angiopoietins 1-4 (Ang1-4), whose activities are mediated through

Constantinos M. Mikelis

constantinos.mikelis@ttuhsc.edu

1 Department of Pharmaceutical Sciences, School of Pharmacy, Texas Tech University Health Sciences Center, 1406 S. Coulter St., Amarillo, TX 79106, USA the tyrosine kinase receptors Tie1 and Tie2 [1-3]. Another important component of this pathway is vascular endothelial-protein tyrosine phosphatase (VE-PTP) [4]. The Ang/ Tie pathway controls growth, survival and maturation of endothelial cells [1, 5-7], with the best characterized ligands of this family being angiopoietin-1 (Ang1) and angiopoietin-2 (Ang2) [2, 4, 5]. Since Ang1 and Ang2 are the best studied and currently the only targeted ligands, this review focused on Ang1 and Ang2.

Members of the Ang/Tie pathway play important roles in endothelial physiology and have been correlated in the pathology of vascular-related diseases [8-12]. In this review article, we summarize what is currently known on the role of the Ang/Tie pathway in the regulation of retinal and pulmonary vascular functions under steady and diseased states, highlight drug candidates in clinical trials that target the Ang/Tie pathway and identify areas worth exploiting for further research or therapeutic applications. The information presented was obtained by a literature search in PubMed for relevant articles reporting the function of each member of the Ang/Tie pathway, the role of this pathway on the pathophysiology of the included vascular disorders and the clinical trials (completed, ongoing or planned) with drug candidates targeting Ang/Tie members. Information about the clinical trials was obtained from clinicaltrials.gov. 


\subsection{Components of the Ang/Tie Signaling Pathway and Their Role in Vascular Development}

Tie2 is the most highlighted, and thus targeted, receptor in the Ang/Tie family, known for its vascular stabilizing and angiogenic effects $[2,4,13,14]$. Tie2 directly interacts with its ligands, Ang1 [15] and Ang2 [3]. The impact of a protein deficiency in development and function is analogous to its significance on that function. In that respect, the Tie2 receptor is the most important player of the Ang-Tie family: Tie2-deficient mice die between E9.5 [16] and E10.5 [2, 17], due to vessel remodeling defects in the plexus of the yolk sac, the brain and due to severe heart defects [2, 16-18]. Tie2-deficient endothelial cells of the endocardium and the sinus vein remain distant from the surrounding mesenchymal cells. This leads to defects, such as occlusion of a part of the sinus venosus, occluded connections between ventricle and aorta and between the atrium and ventricle of the primitive heart [18]. This disturbed connection of the central aorta from the circulatory system results in subsequent arrest of the circulation and possibly death of the embryo at that stage $[18,19]$. These studies demonstrate that Tie 2 is the principal mediator of the vascular functions attributed to the Ang-Tie signaling family.

Contrary to Tie2, Tie1 does not regulate the heart structure, but the vascular network and integrity of the vasculature, with early evidence during lung development, since Tie1-deficient pups died immediately after birth due to breathing difficulties. At E18.5, the Tie1-/- embryos exhibited hemorrhages at the tail, toes and internal organs and subcutaneous edema at the head and back. The edema was attributed to a lack of vessel integrity at the site of leakage and not an abnormal heart function, as seen in the Tie2- and Ang1-deficient mice. The integrity of the blood vessel endothelial cells was compromised at the site of hemorrhage, with increased erythrocyte extravasation [2]. In another study, Tie1-deficient mice exhibited increased number of vascular segments resulting into a dense and more complex vascular network throughout the embryo [18]. In both studies, there was decrease in the size of the alveoli and increased erythrocyte extravasation, supported by the extended intra- and transcellular holes of the intestinal vascular plexus of the mucosa [2, 18].

Ang1 binding induces the auto-phosphorylation of the Tie2 receptor on endothelial cells, through which it elicits its vascular stabilizing effects [15, 20]. Ang1 elicits its activity acting either directly on the endothelium or via the recruitment of peri-endothelial supporting cells [15, 19, 20]. The main downstream mediators of endothelial cell survival upon Ang1 binding to Tie2 are phosphatidylinositol 3'-kinase (PI3K) and Akt [21], which inhibit Smac and caspase $-3,-7$ and -9 activation [22]. PI3K is also responsible for Ang1-induced angiogenic potential via plasmin and metalloproteinase-2 (MMP2) secretion [13], while other mechanisms, such as reactive oxygen species (ROS) play regulatory role [23]. Ang1-induced upregulation of the Kruppel-like factor 2 (KLF2) and the Notch ligand delta-like 4 (Dll4) determines vascular quiescence [24, 25] (Fig. 1). In terms of vascular permeability, Ang1 counterbalances the inductive effect of vascular endothelial growth factor (VEGF) [26, 27] in multiple levels: Ang1-induced Akt activation blocks VEGF-induced permeability [28], while Ang1-driven Src sequestration via mDia, a downstream RhoA effector, deprives the ability of VEGF to phosphorylate and internalize VE-cadherin, and thus induce paracellular permeability [26].

With Ang1 being the main Tie2 ligand, Ang1 deficiency resembles the impact of Tie2 deficiency, and also leads to embryonic lethality, although the difference in the death stage denotes that the impact of its deficiency is less severe than the one observed in Tie2. In Ang1-deficient mice, no newborn pups were found and examination of the pups at early embryonic stages revealed gross abnormalities by E11 and death by E12.5. Similar to the Tie2-deficient mice, Ang1-deficient mice exhibited defects in the vascular endothelium of the developing heart with an immature endocardium. Whole-mount analysis using PECAM1 revealed an immature and less intricately folded endocardium in Ang1deficient embryos as early as E9.5 [19].

Contrary to Ang1, Ang2 mainly inhibits vascular stabilization by blocking Ang1-induced Tie2 phosphorylation [3]. Later studies demonstrated that Ang2 has a contextdependent role, since it has also been shown to phosphorylate Tie2, although not with the same strength as Ang1, inducing endothelial cell functions [29, 30] (Fig. 1). Ang2 is expressed by the endothelial cells, stored in the WeibelPalade bodies, and released in hypoxic and inflammatory conditions, including tumors [31-33]. In pathological conditions, Ang1-Tie2 signaling is inhibited with a subsequent inhibition of PI3K/Akt activation and induction of FOXO1 activation, which leads to Ang2 activation and compensation for Ang 1 activity, increasing Tie 2 activation and angiogenesis [34]. The context-dependent role of Ang2 is further determined by the presence of Tie1 in inflamed or diverse endothelial beds (i.e. lymphatic), where Tie 1 cleavage leads to the switch of the Ang2 activity from agonistic, under normal conditions, to antagonistic [34-37].

Unlike the other members of the Ang/Tie pathway, Ang2 is dispensable for embryonic vascular development, as shown by the normal eye vasculature of Ang2-deficient embryos but is necessary for lymphatic vessel development. Mice lacking Ang2, although initially normal at birth, died after 2 weeks due to severe chylous ascites and lymphatic dysfunction. Furthermore, post-natal examination revealed dramatic defects, due to deficient regression of the hyaloid 
Fig. 1 Schematic representation of the members of the Ang/Tie signaling pathway, their determining interactions in vascular diseases, and the drug candidates targeting each. Arrows in black demonstrate the vascular stabilizing effects induced by Ang1-induced Tie2 activation. Arrows in red demonstrate the destabilizing vascular effect, caused by Ang2 binding to Tie2 or VE-PTP interaction. Current pharmaceutical approaches include intracellular (razuprotafib) or extracellular (ARP-

1536) VE-PTP inhibition, Ang2 blockade (faricimab, nesvacumab, ABTAA, LY3127804) and Tie 2 activation (vasculotide, MAN-01, AV-001). Tie1 is also a mediator of the pathway, but not currently targeted

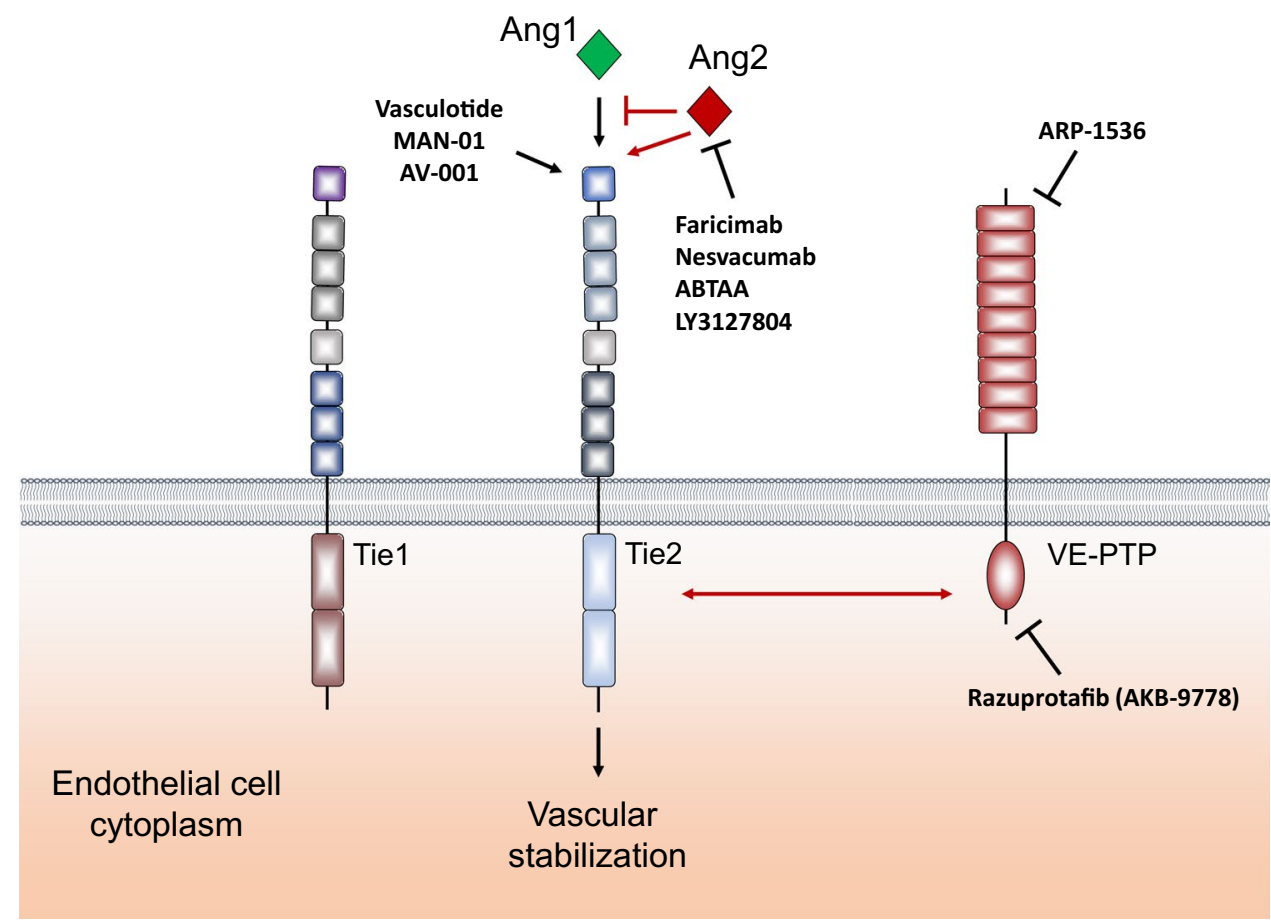

vasculature in the Ang2-deficient mice. In addition, these retinas were peripherally avascular at P10 with a rudimentary superficial vascular plexus to the central retina. These retinal defects were not corrected at later stages but developed secondary pathologic angiogenesis. Substitution of the Ang2 gene with Ang1 rescued the lymphatic defects found in Ang2-deficient mice but did not rescue the defects in vascular remodeling in the neonatal eye [38]. Similar or worse features than the Ang1-deficient or Tie2-deficient mice were observed with Ang2 overexpression, which led to embryonic lethality at E9.5-10.5 [3]. The severe phenotype upon Ang2 overexpression, but not deficiency, along with the similar phenotype upon Tie 2 deficiency go along with an inhibitory role of Ang2 on Tie2 signaling for the vascular functions and further emphasize the context-dependent role of Ang2.

As mentioned above, the features observed in Ang1deficient mice were similar (although not as severe) to Tie2 deficiency or Ang2 overexpression, since Ang1-deficient mice died by E12.5 while the Tie2-deficient and Ang2overexpressing mice died between E9.5-E10 [3, 16, 19]. Although Tie2-deficient mice die earlier, their phenotype presents similarity with the Ang1-deficient mice, which could be attributed to the similarity of their expression patterns during embryonic development. It is noteworthy that the Ang1-deficient mice exhibited reduced levels of Tie2 mRNA [19]. The dominant role of Tie 2 is easily conceivable due to the severity of the phenotype upon Tie2 deficiency. The lethality or defects seen in Ang2 overexpression can be attributed to its antagonistic effect on Tie2 (thus mimicking Tie 2 or Ang1 deficiency phenotypes). The impact on lymphatic endothelium upon Ang2 and Tie1 deficiency demonstrates potential similarities in the lymphatic function, despite the diverse developmental stages. The expression patterns of the other components in this context would be worth investigating.

The Tie2:Tie1 ratio seems to be a reliable determinant of Tie 2 activation [39, 40]. The extracellular domains of Tie 1 and Tie 2 can be cleaved by different agonists, representing an alternative mechanism of cellular responses to stimuli. The extracellular domain of Tie 1 is released under basal conditions in vivo by the activity of MMPs, driven by certain growth factors, such as VEGF, tumor necrosis factor- $\alpha$ (TNF- $\alpha$ ), by phorbol myristate acetate (PMA), or shear stress [41-44]. The cleaved extracellular domain of Tie 1 was shown to bind to Tie2, mediating Tie2 signaling [45], while the intracellular domain, although it cannot induce signaling, interacts with signaling mediators [46]. Similar to Tie1, cleaved Tie 2 forms are normally detected in biological fluids, endothelial cell supernatant, and in animal models of pathological conditions, such as the hindlimb ischemia model. PMA, VEGF and MMPs are the mediators, regulated by the p38 and PI3K pathways [47-49]. Later, the activity of PMA on Tie 2 cleavage was identified to be mediated by MMP-14, which was not regulated by $\mathrm{p} 38$ and PI3K, but by protein kinase $\mathrm{C}(\mathrm{PKC})$ and extracellular signal-regulated kinase (ERK) signaling [49].

Another important component of the Ang/Tie pathway is vascular endothelial-protein tyrosine phosphatase (VEPTP). VE-PTP function involves the regulation of the Tie2 receptor by binding to its cytoplasmic domain, reducing 
its phosphorylation and inhibiting its physiological functions [50-52] (Fig. 1). VE-PTP associates with Tie-2 and VE-cadherin and is pivotal for vascular maintenance and remodeling [53]. Since VE-cadherin is a binding partner of VE-PTP, it has been demonstrated that the stabilizing effect of VE-PTP on endothelial junctions is mediated via its interaction and inhibition of VE-cadherin phosphorylation, independent of Tie2. VE-PTP is required for endothelial cell contact integrity and dissociation from VE-cadherin is triggered by VEGF [54, 55].

Gene deletion or disruption of VE-PTP caused embryonic lethality, vascular remodeling defects, and enlargement of vascular structures in extraembryonic tissues [53, 56, 57]. There is a functional interplay between Tie2, VE-PTP and VE-cadherin: VE-PTP blockade-driven enlargement of cord structures was ameliorated in Tie2-null mice, supporting the notion that VE-PTP controls vascular remodeling by regulating the activation of Tie-2, a mechanism involving Ang1 but not Ang2 [53]. VE-PTP inhibition stabilizes endothelial cell contacts through activation of Tie 2 both in vitro and in vivo [58]. The vascular-stabilizing effects of VE-PTP, were attributed to its interaction with VE-cadherin [55, 59]; however, these effects were instead independent of the cadherins, but dependent on Tie2-driven activation of Rap1 and Rac1 [58]. Although the factors regulating the interplay have not been clearly delineated, cell confluency seems to be a determining factor on the potential compensatory role of VE-cadherin on the effect of Tie2 upon VE-PTP inhibition [58]. The above demonstrate the role of VE-PTP in the Ang/Tie system: in addition to regulating the function of Tie2, VE-PTP also has a functional interplay with other permeability regulators, such as VE-cadherin.

The expression template of the Ang/Tie family members has revealed information regarding their role and mode of activity. At E8.5 and E9.5 Tie2 is expressed in the endothelial cells at the endocardium, the sinus venosus and mesenchymal vessels, while in E12.5 it is expressed in the heart, in large embryonic vessels, intersomitic plexus and vessels of the developing organs. Ang1 bears similar expression pattern with Tie2 [19]. It is expressed in both embryonic days in the surrounding mesenchymal and smooth muscle cells surrounding the blood vessels, denoting paracrine signaling $[3,18]$. In mid-gestational embryogenesis Ang1 is expressed in mesenchymal and smooth muscle cells in neural tissues, the somites, and the lung [3]. At the same developmental stage, Ang2 is not detected in the developing heart, but in the endothelium lining the big vessels. In the adult, Ang2 is not expressed in the heart and vessels, but in vascular remodeling sites, such as ovary, placenta, and uterus [3]. Ang2 expression in the resting endothelium is limited, and it is induced upon activation. It remains stored at WeibelPalade bodies to be released upon stimulation $[33,60]$. VEPTP expression is endothelial-specific [51, 57]; at E10.5 it is strongly expressed at the heart, aortic sac, outflow tract, dorsal aortas, and arteries, but less in the veins. At E15.5, a strong expression in the heart valves was obvious, while the expression template remained similar [57]. However, the physiological expression patterns of the Ang/Tie members are often differentiated in pathological conditions. As an example, in angiosarcomas, endothelial-related tumors, the majority of the cases correlate with strong expression of Ang1, Tie1 and Tie2, whereas Ang2 expression was more heterogeneous and correlated with vasoformative architecture. Anti-Tie2 treatment led to growth delay, with a more pronounced effect when combined with VEGFR inhibition $[61,62]$.

\section{The Ang/Tie Pathway in the Retinal Vasculature}

\subsection{Retinal Vascular Function}

The eye has high metabolic needs, facilitated by the integrated activity of three vascular systems, the choroidal, the hyaloid and the retinal vascular systems, which drain from the ophthalmic artery, a branch of the internal carotid artery [63-66]. The choroid and the hyaloid vessels support the retinal oxygenation during the initial developmental stages of the eye. The hyaloid vascular network is transient and regresses at the final stages of retinal development, whereas the choroidal vascular network increases in density to form a dense vascular network around the outer layer of the optic cup [66]. The choroidal system consists of the choroidal capillaries, which are derived from the ciliary arteries, originating from the ophthalmic artery supplying the outer retina mainly the photoreceptor cells [63-65]. Although the development of the choroid vessels is less studied than the retinal vessels, their development seems to depend on the presence of the retinal pigment epithelium [66]. The limited initial thickness of the retina allows efficient diffusion of oxygen from the choroid, but as its thickening increases, it induces the formation of the retinal vessels [67]. The retinal vessels are organized in three layers restricted in the retina [66]. The retinal system consists of the central retinal artery and corresponding vein both of which supply and drain the inner retina [63-65]. In the adult, the blood supply is separated into the retinal and the choroidal system [63-65]. Retinal vessels are autoregulated to optimize blood supply to meet the metabolic needs of the retina [63].

The development of the vascular network of the retina produces a network of vessels that meet the high metabolic demands of the healthy adult retina. For proper retinal development and function, a tight balance needs to be maintained in the vessel formation, especially due to the fact that the retina has the highest metabolic demand of 
any tissue in the body $[63,68]$. Angiogenesis is an important process in both physiological vascular development and pathological neovascularization. Abnormal ocular angiogenesis occurs in a broad spectrum of eye disorders, such as retinopathy of prematurity, diabetic retinopathy, neovascular age-related macular degeneration (NV-AMD), neovascular glaucoma, and corneal neovascularization [69, 70].

The function of the Ang/Tie pathway in physiological and pathological retinal vascular development has been well demonstrated. In Ang2-deficient mice, the delayed and incomplete development of the superficial vascular bed and the complete absence of the intermediate and deep vascular beds of the retina were reported, processes that rely on vasculogenesis and angiogenesis, respectively [71]. Moreover, the hyaloid vessels would not regress, mimicking the infant phenotype of persistent fetal vasculature, driven by a relatively common congenital abnormality [38, 71-73]. Although at birth the retinal vasculature was not affected, there were severe defects in hyaloid vasculature regression at subsequent stages of vascular remodeling. Instead of a well-developed superficial vascular network covering the entire surface of the retina, the retinal vasculature of Ang2-deficient P10 mice was superficial and restricted to the central retina, a condition that was not ameliorated in the few mice that survived to P25. Instead, the hyperplastic hyaloid vasculature expanded in the retina, as a secondary pathologic angiogenic process [38]. An alternative splice variant of Ang2, named ANGPT2443, is a less oligomerized form and competitive antagonist of Tie2 activation. Forced expression of ANGPT2443 in a knock-in murine model impaired venous development in the retina [74]. Tie2 was identified as an essential component for venous specification and maintenance. Tie2-deficient mice exhibited a significant decrease of retinal vascularization and venous degeneration, accompanied by the formation of hemangioma-like vascular tufts. This Tie2-dependent venous vasculature maturation and maintenance was controlled via the Tie2 downstream signaling mediators PI3/Akt. PI3/Akt activation regulated COUP-TFII protein stability [17]. Contrary to Tie2 and Ang2, inducible Ang1 expression during retinal vascular development did not alter vascular density and had no effect on established retinal or choroidal neovessels. However, simultaneous Ang1 expression with VEGF suppressed VEGF-induced neovascularization, demonstrating stage-dependent Ang 1 effects [75]. The vascular alterations upon deficiency or overexpression of the Ang/Tie proteins highlight the significance of this pathway in retinal vascular development.

The VEGF/VEGFR pathway is the major target in the current standard of care therapies of retinal pathologies. VEGFR2 is well established as the earliest marker of developing endothelial cells and significant data have delineated its role in retinal vessel sprouting and the interaction among endothelial cells and other cell types, such as astrocytes [76-78]. The current first line of anti-angiogenesis drugs for retinal vascular diseases target the VEGF pathway through currently approved drugs, such as macugen, bevacizumab, ranibizumab and aflibercept.

- Macugen (Pfizer Inc.), formerly pegaptanib sodium, is an anti-VEGF 27 nucleobase-long RNA aptamer targeting VEGF165. It was the first FDA-approved anti-angiogenic therapy indicated for the treatment of NV-AMD. The molecule was first produced by the systematic evolution of ligands by exponential enrichment (SELEX), an approach for aptamer selection [79]. It binds to the hairpin binding site of VEGF165 and inhibits its downstream signaling [80].

- Bevacizumab (Avastin, Genentech Inc.), is a full length humanized monoclonal antibody that specifically binds to VEGFA, thereby inhibiting the process of angiogenesis, resulting in regression of existing micro vessels, normalization of surviving mature vasculature, and inhibition of vessel growth and neovascularization [81-83].

- Ranibizumab (Lucentis, Genentech Inc.) is a cleaved Fab fragment of bevacizumab with a similar mechanism of action, but enhanced affinity [84-87]. Because bevacizumab was a full-length antibody, there were concerns about using it as intravitreal injections as it was associated with a low but significant risk of acute intraocular inflammation and sporadic cases of blindness worldwide. In addition, the fate of intravitreally injected full-length antibodies is not yet fully understood $[88,89]$. This makes ranibizumab a more preferred option for intravitreal injections considering that it better neutralizes VEGF at low concentrations, maintains efficacy longer, and has a higher retinal penetration and potency. However, bevacizumab is still preferred, due to low cost and first-to-market availability.

- Aflibercept (Eylea, Regeneron Pharmaceuticals), also known as VEGF Trap-eye (VTE), is a recombinant fusion protein that binds to members of the VEGF family. It has been shown that effective blockade of VEGF signaling pathway involves preventing binding of VEGF to its normal receptors by administering this decoy-soluble receptor [90-92]. Unlike the monoclonal antibodies bevacizumab and ranibizumab, aflibercept was produced by fusing the first three Ig domains of VEGFR1 to the constant region $(\mathrm{Fc})$ of human IgG1, hence the name VEGFTrap [92]. By maintaining high affinity and extending the in vivo half-life, the improved VEGF-Trap used in aflibercept provided better pharmacokinetic properties.

Although most of these therapies were originally developed as anti-tumor therapies, they are now approved for the 
treatment of several retinal vascular disorders. However, the time- and tissue-dependent interplay among VEGF and other angiogenesis mediators, defines the final retinal vasculature growth and function, as well as the potential for development of retinal vascular diseases [76-78]. Angiopoietins are among the important angiogenesis mediators, as the Ang/ Tie pathway controls the growth, survival, and maturation of endothelial cells [5]. Several mouse retinal models have highlighted the role of the Ang/Tie proteins in retinal vascular development and maintenance [17, 93, 94] (Fig. 2). It is, therefore, necessary to highlight the role of the Ang/ Tie pathway as a therapeutic possibility for retinal vascular pathologies. Below, we discuss some of the common retinal disorders, highlighting the role of the Ang/Tie pathway in the pathology of each disease and the drugs targeting this pathway that are currently under clinical evaluation.

\subsection{Retinal Vascular Disorders}

Retinal vascular disorders are associated with a defect in the blood vessels of the eye and are mostly linked to existing vascular diseases, such as hypertension, cholesterolemia, diabetes and conditions that cause atherosclerosis. These diseases are multifactorial, and they influence a number of biological processes in cells and tissues [95]. Among the most common retinal vascular disorders are age-related macular degeneration, diabetic retinopathy, hypertensive retinopathy, and vascular occlusions (retinal vein occlusion, central retinal artery occlusion, branch retinal occlusion) [96-100] (Fig. 2).

\subsubsection{Age-Related Macular Degeneration}

Age-related vascular degeneration (AMD) is a common condition and a leading cause of visual impairment and severe vision loss for people aged $\geq 50$ years. It is an acquired disease of the functional portion of the retina due to late-onset neurodegeneration of the photoreceptor-retinal pigment epithelial complex, leading to progressive visual impairment [101]. Due to the multifactorial nature of AMD, the knowledge on its pathophysiology and the underlying mechanisms are still expanding. AMD has been characterized with extracellular matrix dysfunction and dysregulation of the complement, lipid, angiogenic and inflammatory pathways [102]. NV-AMD, a form of AMD, is characterized by the formation of choroidal neovascularization, an ingrowth of abnormal blood vessels from the choroid through Bruch's membrane into the sub-retinal pigment epithelium or subretinal space [103]. These abnormal blood vessels demonstrate abnormal leakiness of fluids and blood into the retina, causing vision distortion and loss of central vision [102, 104]. AMD treatment focuses on the inhibition of angiogenesis, via the most common target, vascular endothelial growth factor-A (VEGF-A) due to its excessive expression in the hypoxic regions [101, 102]. However, this has its limitations as the therapy requires periodic and repetitive administration [105], raising a concern for long-term anti-VEFG therapy, which can eventually lead to retinal pigment epithelium (RPE) damage, photoreceptor degeneration, and permanent vision loss [106-108] and instances of resistance, non-response and recurrence of the pathology [109], implying the involvement of factors other than VEGF.

The Ang/Tie signaling pathway is a known mediator of AMD vascular deficiencies $[110,111]$. The Ang/Tie2 pathway maintains vascular integrity, and negative regulators of this pathway are potential therapeutic targets for these diseases. Tie 2 activation by intraocular injection of an antibody for VE-PTP, a negative regulator of TIE2 activation, suppressed ocular neovascularization, therefore stabilizing retinal and choroidal blood vessels [112].
A

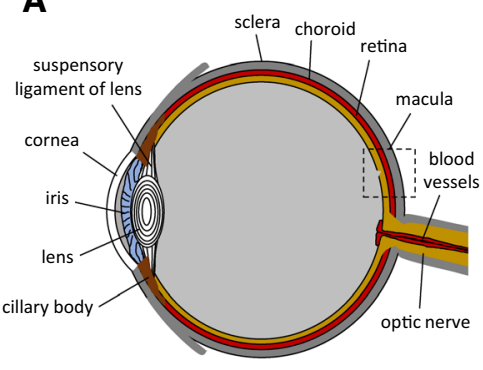

B

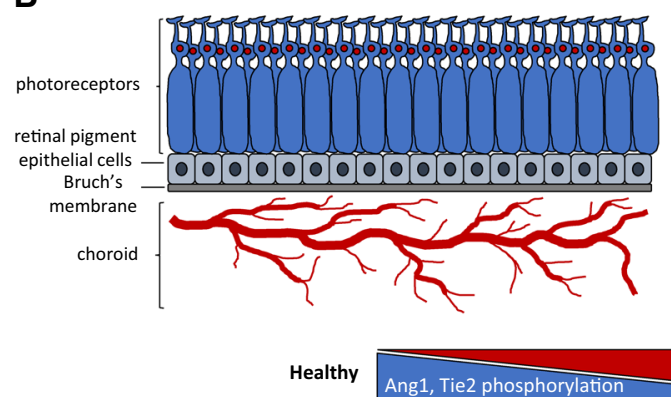

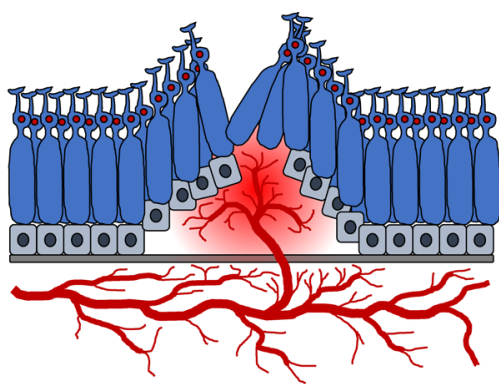

VEGF, Ang2

AMD (compromised Bruch's membrane) Proliferative Diabetic Retinopathy Macular Edema
Fig. 2 A Schematic representation of the transverse cross-section of the eye. The parts and characteristic structures of the eye are depicted. B Abnormal angiogenesis of the choroid vessels during retinal vascular disorders. The abnormal, newly formed vessels disrupt the integrity of Bruch's membrane, alter the structure of the reti- nal pigment epithelial cells and photoreceptors and cause subretinal hemorrhage, fluid accumulation and visual impairment. The severity of the retinal vascular disorders correlates with the levels of VEGF, Ang2, and VE-PTP and inversely correlates with the levels of Ang1 and Tie 2 phosphorylation 
Increased Ang2 levels have been observed in the retina during the angiogenic development of the deep retinal capillaries and during pathologic angiogenesis, showing a temporal and spatial correlation of Ang2 activity in the retina $[72,112]$. Contrary to Ang2, Ang 1 has a protective role in the retina during neovascularization, but no effect in adult or established vasculature. Overexpression of Ang 1 in the retina during retinal vascular development inhibited neovascularization but did not affect the established retinal or choroidal vasculature, and when its expression was induced along with VEGF, it antagonized the activity of VEGF, preventing neovascularization and retinal detachment $[75,113]$. These observed vascular outcomes of the Ang/Tie proteins in the retina pinpoint the significance of this pathway in retinal vascular development and form the basis for further analysis of Ang/Tie vascular mechanisms.

\subsubsection{Diabetic Retinopathy}

Diabetic retinopathy is a pathological condition that can cause vision loss and blindness in diabetic patients. It is a major complication of diabetes mellitus and a leading cause of visual loss in working-age population. Diabetic retinopathy is divided into non-proliferative and proliferative diabetic retinopathy. Proliferative diabetic retinopathy is characterized by neovascularization, during which, abnormal vessel growth and bleeding occurs into the vitreous, and tractional retinal detachment can happen [114]. The most common cause of vision loss in diabetic retinopathy is diabetic macular edema, described below, a condition characterized by swelling or thickening of the macula due to accumulation of fluid, as a result of the breakdown of the blood-retinal barrier [114, 115]. Diabetic retinopathy is considered a microvascular complication, therefore most therapeutic interventions aim at the management of microvascular complications, such as intravitreal pharmacologic agents, laser photocoagulation and vitreous surgery. Among the intravitreal pharmacologic agents, the intravitreal administration of anti-VEGF agents is the main therapy for both early and advanced stages of diabetic retinopathy [114]. Although these therapies may provide stabilization of visual acuity or visual improvement, there are ocular adverse effects or inadequate responses.

\subsubsection{Macular Edema}

Macular edema is defined as the abnormal increase of fluid in the macula, an area at the center of the retina. Although it is a leading cause of major vision loss in diabetic persons and commonly related to diabetic retinopathy, it has other causes, such as branch and central retinal vein occlusion and postoperative inflammation [116, 117]. In macular edema, the fluid buildup, which is due to a break in the blood-retinal barrier, causes the macula to swell and thicken, which distorts vision. Oxidative stress, a characteristic feature in diabetes, vascular retinal diseases and lung or heart diseases, upregulates multiple cytokines and chemokines, such as VEGF, angiopoietins, tumor necrosis factor, interleukins (ILs) and matric metalloproteinases, that lead to the breakdown of the blood-retinal barrier [117-119]. Intra-ocular injections of anti-VEGF have become the most preferred therapeutic option for macular edema and have been shown to be effective in vision improvement and prevention of vision loss. However, being a feature of diabetic retinopathy, macular edema is an inflammatory disease with many cytokines and chemokines involved in the process [119, 120]. Consequently, many macular edema patients do not show complete response to anti-VEGF drugs, despite multiple intravitreal injections, with ephemeral remission and non-complete resolution of fluid [119, 121, 122]. Considering that VEGF may not be the predominant mechanism of vessel permeability in macular edema [122], there is a need for supplemental treatments that might offer better therapeutic efficacy.

Several systemic conditions associated with vascular leakage correlate with elevated levels of serum Ang2 [123-125]. In macular edema patients, the vitreous levels of Ang2 are significantly augmented. Moreover, Ang2 inhibition decreased vascular permeability and inflammation in the retina, by blocking leukocyte adhesion to the vessel walls, inhibiting the expression of ICAM-1, CCL2 and CCL5, and decreasing monocyte trafficking and microglia activation in the retina $[119,126]$. These studies on Ang2 in retinal diseases, along with the extensive knowledge of the role of the Ang/Tie pathway in retinal vascular development, as mentioned above, have led several Ang/Tie pathway-targeting drugs into clinical trials. Most have demonstrated promising results, especially in combination with VEGF inhibition.

\subsection{Drugs in Clinical Trials Targeting the Ang/Tie Pathway for Retinal Vascular Disorders}

Several anti-angiogenesis drugs have been Food and Drug Administration- (FDA)-approved for the treatment of retinal vascular disorders. Since most are monotherapies for the VEGF signaling, as mentioned above, they have shown limitations. Below, we highlight novel drug candidates, classified according to the target molecules, currently in clinical trials (Fig. 1) that target Ang/Tie signaling pathway for vascular disorders in comparison with anti-VEGF inhibition or, sometimes, concomitantly with anti-VEGF activity. 


\subsubsection{Tie2 Receptor}

MAN-01: Given the known activity of the Ang/Tie signaling in lymphatic vessels $[5,127]$, the development of Tie2-targeting drug candidates for lymphatic vascular ocular diseases was expected. MAN-01 or MAN 01 (Mannin Research; Q BioMed) is a small molecule modulator of Tie2 and is an example of a drug candidate targeting this pathway. It targets the Schlemm's canal, a circular lymphatic-like vessel in the eye, the obstruction of which leads to glaucoma $[128,129]$. Although no significant information about the mechanism has been released, administration of MAN-01 in mice has provided positive results [130] and the intention to submit an investigational new drug (IND) application to the FDA has been announced [131].

\subsubsection{Vascular Endothelial-Protein Tyrosine Phosphatase (VE-PTP)}

Razuprotafib (AKB-9778): Razuprotafib (AKB-9778) (Aerpio Pharmaceuticals) is a small molecule inhibitor of VEPTP. AKB- 9778 acts by binding and inhibiting the intracellular catalytic domain of VE-PTP and stabilizing endothelial junctions [58, 112]. Subcutaneous razuprotafib was an investigational drug for treatment of diabetic vascular complications, such as diabetic macular edema, inflammatory leakiness, diabetic nephropathy, and open angle glaucoma [42, 64-67]. In the Phase 2a TIME clinical trial for diabetic macular edema, razuprotafib (AKB-9778) was patient selfadministered via a subcutaneous injection, similar to insulin. The combination of AKB-9778 with ranibizumab enhanced the reduction of diabetic macular edema compared to ranibizumab alone. In the Phase 2b TIME clinical trial, AKB9778 was administered once or twice daily versus placebo in patients with non-proliferative diabetic retinopathy. This study, completed in March 2019, although it failed to meet its primary endpoint on diabetic retinopathy severity score, it noted some improvement in patients treated twice daily for 48 weeks $[132,133]$.

ARP-1536: ARP-1536 (Aerpio Pharmaceuticals) is a humanized monoclonal antibody that targets the extracellular domain of VE-PTP, leading to increased Tie2 activation, similar to razuprotafib, but was administered intravitreally [134]. As previously mentioned, VE-PTP is a phosphatase that binds to both Tie 2 and VE-cadherin. It was shown that antibodies targeting the extracellular domain of VE-PTP induce the endocytosis of the Tie2-bound VE-PTP molecules, reducing the inhibitory effect of VE-PTP on Tie2 and leading to Tie2 activation. VE-cadherin-bound VEPTP molecules are not endocytosed, thus the interaction and the VE-cadherin-mediated functions were not affected by antibodies targeting the extracellular domain of VE-PTP
[53]. However, it should be emphasized that both razuprotafib and ARP-1536 showed greater potential when used in combination with anti-VEGF therapy compared with antiVEGF therapy alone $[132,135]$. According to the current knowledge, inhibiting VE-PTP mainly targets Tie2, as it induces its functionality, recapitulating the action of Ang1. Although the target seems to be optimal, the outcome could be improved if the administration route is modified (i.e. from subcutaneous injection to intravitreal administration for the AKB-9778). The simultaneous targeting of more pathways by combinatorial therapy with current VEGF-based therapeutic approaches, is a preferable option, with expected optimal results.

\subsubsection{Angiopoietin 2 (Ang2)}

Faricimab: Faricimab (Roche), also known as RG7716 or RO6867461, is a bispecific antibody targeting both VEGF-A and Ang2. This bispecific antibody comprises two different heavy chains and two different light chains, from which one binds to VEGF and the other to Ang2. It is a product of the CrossMAB technology that provides correct heterodimerization of two different antigen-binding domains in a single molecule $[109,136]$.

The completed Phase 2 clinical trials of faricimab comprised three studies, the AVENUE, BOULEVARD and STAIRWAY clinical trials [137]. The active comparator in all three studies was ranibizumab (Lucentis ${ }^{\circledR}$ ), a humanized anti-VEGF monoclonal antibody shown to improve visual acuity (VA) in patients with wet AMD in randomized clinical trials $[138,139]$, but with considerably poorer patient outcomes in clinical practice [138]. The AVENUE study was a proof-of-concept study in participants with choroidal neovascularization secondary to AMD and had the following arms: Arm A (intravitreal injections [IVT] of $0.5 \mathrm{mg}$ ranibizumab once every 4 weeks [Q4W]) for 32 weeks, arm B (IVT of $1.5 \mathrm{mg}$ faricimab Q4W for 32 weeks), arm C (IVT of $6 \mathrm{mg}$ faricimab Q4W for 32 weeks), arm D (IVT of $6 \mathrm{mg}$ faricimab Q4W for 12 weeks, $6 \mathrm{mg}$ every 8 weeks until Week 28) and arm E (IVT $0.5 \mathrm{mg}$ ranibizumab Q4W for 8 weeks followed by $6 \mathrm{mg}$ of faricimab Q4W up to Week 32 ), with patients being monitored up to 36 weeks. Although the primary end point over ranibizumab was not met, overall visual and anatomical gains in the faricimab group led to Phase 3 trials as a potential alternative to monthly antiVEGF therapy [140]. The BOULEVARD study was conducted in participants with center-involving diabetic macular edema and included 3 groups: Arm A ( $0.3 \mathrm{mg}$ ranibizumab), arm B (1.5 mg faricimab) and arm C (6 mg faricimab). All groups received IVT of the assigned drug Q4W up to Week 20 and were observed up to Week 36 . As a primary outcome, faricimab provided superior VA gains versus ranibizumab at Week 24, while central subfield thickness reduction, diabetic 
retinopathy severity score improvement, and extended durability also supported the primary outcome [141]. The STAIRWAY study evaluated faricimab compared to ranibizumab for extended durability in the treatment of NV-AMD. The main outcome was the mean change in best-corrected VA at Week 52, and faricimab administration with 16-week and 12-week dosing intervals resulted in maintenance of initial vision and anatomic improvements in comparison to monthly ranibizumab administration [142]. The ALTIMETER trial, an ongoing Phase 2 study for diabetic macular edema is investigating the aqueous humor and multimodal imaging biomarkers in 80 treatment-naïve participants with diabetic macular edema treated with faricimab [137]. The efficacy and safety of faricimab are currently under evaluation in Phase 3 clinical trials for AMD and diabetic macular edema. The YOSEMITE, LUCERNE, TENAYA and RHINE clinical trials have recruited/are recruiting participants for the sham and aflibercept (the active comparator) groups running alongside faricimab. Finally, the Phase 3 clinical trial Rhone- $\mathrm{X}$ is evaluating the long-term safety and tolerability of faricimab on diabetic macular edema [137]. Faricimab is also being evaluated in Phase 3 clinical trials as a therapeutic option for macular edema secondary to central retinal, hemiretinal or branch retinal vein occlusions. The COMINO and BALATON studies will compare faricimab with aflibercept and sham procedure [137].

Faricimab binds Ang2 but has no effect on Ang1 signaling. Unlike Ang 1, with levels that were weakly elevated or even decreased, increased Ang2 levels have been observed in several human retinal vascular diseases, such as wet AMD, diabetic retinopathy, proliferative diabetic retinopathy, and retinal vein occlusion [110]. Moreover, the interplay between VEGF-A, Ang1 and Ang2 has been reported to regulate the endothelial barrier. In addition to the increased secretion of Ang2 by human endothelial cells [143, 144], VEGF-A further elevated Ang2 levels while Ang1 decreased this VEGF-A-induced Ang2 elevation. Treatment with antiAng2 antibody inhibited VEGF-induced barrier breakdown, demonstrating that Ang2 participates in VEGF activity, which was further supported with the application of a bispecific antibody with combined targeting of Ang2 and VEGFA [110]. Under pathophysiological conditions, dual targeting of both VEGF and Ang2 reduced the number of vessel lesions, permeability, retinal edema, and neuron loss [109]. Thus, the effect from dual inhibition is more pronounced than from single inhibition of either factor. The combined targeting of VEGF-A and Ang2 definitely provides an advantage for faricimab, compared to monotherapy and thus has a higher chance of success. The potency of faricimab on VEGF inhibition compared with anti-VEGF approaches should be further evaluated, which could define whether faricimab should be used in combination with current antiVEGF inhibitors, or it could stand as a monotherapy.
Nesvacumab: Nesvacumab or REGN910 (Regeneron) is a fully human immunoglobulin G1 (IgG1) monoclonal antibody that specifically binds and inactivates Ang2 [145]. Although the safety profile of this antibody was acceptable, when tested as a coformulation with aflibercept, it failed to show benefit over aflibercept monotherapy in Phase 2 studies of AMD (ONYX) and diabetic macular edema (RUBY) $[134,145]$.

ABTAA: ABTAA (Ang2-binding and Tie2-activating antibody) is, as the name indicates, an antibody that binds Ang2 and activates Tie 2 signaling. It has not been clinically tested, but a recent study demonstrated that it suppressed choroidal neovascularization and vascular leakiness, regenerated the choriocapillaris and relieved hypoxia in a Tie2 deficiencydriven NV-AMD model. The potency of ABTAA against NV-AMD is promising for a new therapeutic strategy for NV-AMD that overcomes current anti-VEGF side effects [104]. In addition to its effect in NV-AMD, in glaucoma, intraocular ABTAA administration increased Tie2, p-Tie2, Klf4 and Prox 1 (an important factor for Schlemm's canal development and maintenance) levels. The increased Prox1 was Tie2-ERK-dependent although it had no effect on intraocular pressure and Schlemm's canal areas. The activity of ABTAA was mediated by Tie2, since upon Tie2 deficiency the drug was not effective. However, the absence of Ang1 and Ang2 did not hinder the effect of ABTAA, demonstrating the potential presence of other factors mediating Tie2 activity in Schlemm's canal physiology [146], emphasizing an Ang-independent activation of Tie 2 in the lymphatic vasculature. This somewhat contradictory finding could be explained by the potential of the ABTAA to directly trigger Tie 2 activation in HUVEC, but only to a negligible extent [147]. Tie2/Ang2 activity is elevated in the lymphatic endothelial cells due to the lack of VE-PTP in the lymphatic endothelium [148]. Considering the ability of ABTAA to trigger Tie 2 signaling by itself suggests it could share structural similarities with Ang2 and this could explain the observed Ang1/2-independent Tie2 effects in rejuvenating Schlemm's canal. Furthermore, combination of ABTAA with anti-VEGF-A inhibitors would be expected to increase the treatment potency. A similar approach could be implemented with nesvacumab.

Addition of soluble recombinant proteins is an efficient method for protein inhibition. Studies using recombinant fusion proteins consisting of the ectodomain of Tie2 fused to the Fc portion of human IgG1 demonstrated the impact of this receptor in retinal vascular disorders. Tie2 inhibition upon the addition of these recombinant proteins has demonstrated its role in ischemia-induced retinal neovascularization and retinopathy of prematurity $[149,150]$. Overall, the constantly increasing data on the role of Ang/Tie signaling components in retinal vascular diseases has led 
to an analogous, constantly growing interest in novel drug candidates targeting the Ang/Tie pathway, either as a monotherapy or part of combination therapy in retinal disorders, with high clinical potential.

\section{The Ang/Tie Pathway in Respiratory Vasculature}

\subsection{Respiratory Vascular Function}

The development of the pulmonary vasculature and circulation is an intricate process involving sequential differentiation of mesenchymal progenitor cells, formation of the primitive vascular plexus, and vascular remodeling [151]. Lung development requires the coordination of molecular, morphogenetic, and mechanical events within the differentiating respiratory epithelium. Vascular growth factors from the distal lung buds are thought to promote capillary expansion via intussusceptive angiogenesis. Several receptor tyrosine kinase (RTK) pathways have been implicated in these activities, such as VEGF, angiopoietins, ephrins and basic fibroblast growth factor (bFGF) [151, 152], with VEGF playing a crucial role, especially in the early stages of vasculogenesis, and defining overall pulmonary growth $[153,154]$. The Ang/Tie pathway plays defining roles in vascular development; however, knowledge on its specific functions in pulmonary vascular development is limited. An early study by Sato et al 1995, showed that neonates homozygous for Tie1 mutation died immediately after birth as a result of breathing difficulties. These embryos also had smaller hearts, and histological analysis revealed that alveoli failed to expand in their lungs probably as a result of pulmonary edema. To the contrary, Tie 2 deficiency led to embryonic lethality earlier due to vascular network malformations, demonstrating the distinct functions Tie 1 and Tie 2 regulate [2]. The role of this pathway during pathological pulmonary vascular remodeling has been investigated in lung metastasis $[155,156]$ and respiratory disorders, such as acute lung injury, acute respiratory distress syndrome, and pulmonary edema [11, 12, 157] (Fig. 3). Below, we summarize the role of the Ang/Tie pathway in acute respiratory distress syndrome and pulmonary edema with a brief emphasis on COVID-19 and we highlight clinical trials of drugs targeting the Ang/Tie pathway in pulmonary vascular disorders.

\subsection{Respiratory Vascular Disorders}

Pulmonary vascular disease (PVD) refers to a group of conditions affecting the blood vessels in the lungs. This is due to poor or abnormal blood flow between the heart and lungs and disease progression will eventually affect the rest of the body.

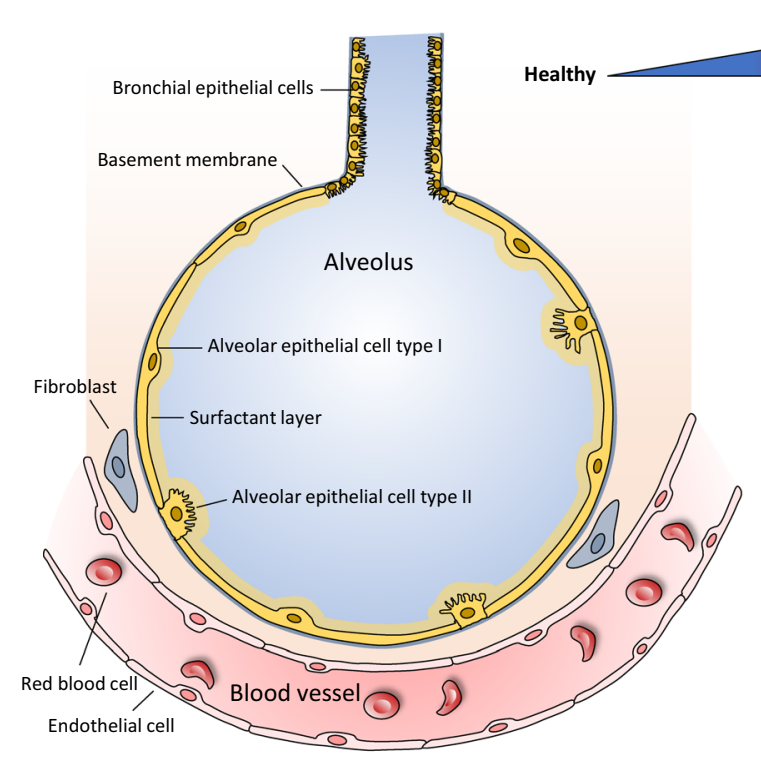

Fig. 3 Schematic representation of the alveolus during healthy conditions versus pulmonary vascular disorders. The pulmonary vascular disorders are characterized by disruption of the endothelial and epithelial integrity, with increased levels of edema fluid invading the

intra-alveolar space. The surfactant function is inhibited, and hyaline membrane formation is initiated. In severe cases, this leads to alveolar flooding and collapse leading to hypoxemia 


\subsubsection{Acute Respiratory Distress Syndrome/Acute Lung Injury}

Acute Respiratory Distress Syndrome (ARDS) is a common cause of acute respiratory failure. It is a more severe form of acute lung injury. ARDS is characterized by alveolar-capillary barrier failure, which results in non-cardiogenic pulmonary edema and life-threatening hypoxemia $[157,158]$. In terms of therapy, there are limited options for ARDS for a number of reasons, one of which is the lack of confirmed biomarkers with a proven causal role in ARDS that can be used to improve clinical trial efficiency [157]. Ang2 has been implicated in endothelial activation and induction of permeability, important factors in the pathophysiology of ARDS [11, 157, 159]. Several studies have supported this role of Ang 2 in disruption of barrier integrity [3] and in the potentiation of lung injury [160, 161]. Although plasma Ang2 has been strongly associated with ARDS, the hypothesis that plasma Ang2 can be a causal factor in ARDS development exists, without being verified so far. Overall, targeting plasma Ang2 could be a promising therapeutic option in the treatment of ARDS, considering the lack of direct targets or therapeutic options in the treatment of this condition.

\subsubsection{Pulmonary Edema}

Pulmonary edema is a condition characterized by an abnormal build-up of fluid in the extravascular tissues of the lungs. Pulmonary edema is not a disease itself but rather an indication of one. There are two major mechanisms involved in the formation of pulmonary edema: i) increase in capillary hydrostatic pressure, which causes high-pressure edema or cardiogenic edema, and ii) increase in vascular permeability, causing permeability edema [162]. Permeability edema is characterized by increased leakiness of the pulmonary vascular endothelium and alveolar epithelium to water and large molecules, such as proteins [162]. The Ang2-Tie2 signaling axis contributes to the endothelial barrier mechanisms, therefore regulating vascular permeability $[5,12,60,163]$. The participation of the Ang/Tie pathway in the pathophysiology of several pulmonary vascular conditions, such as pulmonary edema, pneumonia, and sepsis, as well as the regulatory activity of Ang2 on vascular responsiveness upon activity of inflammatory cytokines [60], imposes the emphasis of angiogenic proteins, such as the angiopoietins, as potential therapeutic targets.

\subsubsection{Pulmonary Arterial Hypertension}

The members of the Ang/Tie family have been correlated with the pathophysiology of pulmonary arterial hypertension $(\mathrm{PAH})$, with often contradictory results. A reciprocal relationship between the bone morphogenetic protein (BMP) signaling and Ang1 expression was initially described, as Ang1 expression was increased in the lung tissue upon systemic-to-pulmonary shunting [164, 165]. Ang1 upregulation and the subsequent elevated Tie2 phosphorylation blocked BMPR2-driven signaling in human pulmonary artery endothelial cells, directly correlating with the severity of the disease [166]. This was contradicted not long after, as Ang1 administration was shown to prevent PAH development by preventing microvessel regression and destruction [167]. Combination of upregulation of Ang1 with VEGF or hepatocyte growth factor (HGF) promoted vascular stability, alleviating PAH symptoms in the monocrotaline-induced rat PAH model [168]. In other studies, no causal or biomarker role was supported for Ang1 [169-171]. Instead, Tie2 levels were induced in patients with idiopathic or systemic-sclerosis PAH, but not PAH associated with other diseases [169, 172, 173]. Moreover, despite the Ang1 and Tie2 overexpression in plexiform lesions, a PAH feature [174], the use of transgenic mice supported the notion of a protective role of Ang1 and Tie2 for PAH [175].

The role of Ang2 in PAH is not straightforward either: although no statistical increase was detected in Ang2 levels in an initial PAH study [169], these levels were significantly increased in patients with idiopathic/familial PAH, correlated with cardiac index and consisted an independent risk factor for mortality [176, 177]. Prostacyclins are known to improve symptoms in scleroderma-associated $\mathrm{PAH}$, and treprostinil, a stable prostacyclin analog, improved $\mathrm{PAH}$ symptoms with an associated decrease in plasma Ang2 levels [176]. However, arterial hypertension was among the side effects of concomitant VEGF-A and Ang2 blockade by vanucizumab, a bispecific antibody targeting both, in patients with solid tumors [178], although the Ang2 impact is not clearly defined. In PAH patients, Ang2 levels were recently associated with higher right atrial pressure, an established risk factor for mortality [179]. These contradicting findings on the involvement of the Ang/Tie pathway in PAH call for further investigation on the Ang/Tie-PAH interplay. Targeting the Ang/Tie members in vitro and in vivo would be necessary to delineate the potential specific roles of this pathway in PAH.

\subsubsection{Asthma}

The role of the Ang/Tie2 family in asthma, a chronic inflammatory disease characterized by angiogenesis and microvascular remodeling [180], is still not fully elucidated. Asthma 
is partly mediated by recruitment of eosinophils, which express Tie2, and secrete lipid mediators and cytokines. Eosinophil migration was driven by Ang1, highly expressed in asthmatic airways, and blocked by Ang2 [181, 182], reproducing their known competitive roles in the endothelium. Several studies have reported alterations of Ang1 and Ang2 levels in the sputum of asthmatic patients. Ang2 levels were induced in the sputum of asthmatic patients in most studies and thus Ang2 has been proposed as an index of exercise-induced bronchoconstriction in asthma, while Ang1 expression is not consistent [183-187]. Serum Ang1 levels and Ang1/Ang2 ratio were decreased in asthmatic children, with Ang2 serum levels remaining stable [188]. However, in adults, serum Ang1 levels were increased, while changes of Ang2 serum levels were not consistent in asthmatic conditions [189, 190]. In an ovalbumin asthma mouse model, Ang1 levels were decreased, similarly to Tie2 activation, and its administration ameliorated the inflammatory response [191]. However, in the same model, Tie 2 expression was detected not strictly in the endothelial cells, as is the case for steady state conditions, but also in airway epithelial cells and macrophages. This was also the case for Ang2, demonstrating that Ang2 and Tie 2 ectopic expression and expression levels correlate with the severity of airway remodeling [192]. In line with the mouse data, Ang1 administration via aerosolized mesenchymal stem cells improved asthmarelated airway inflammation in rabbits [193]. Studies of single nucleotide polymorphisms (SNPs) for the Tie2 gene $(T E K)$ and their association with asthma susceptibility and severity also have contradictory results [194, 195], demonstrating that the roles of the Ang/Tie2 family members are not yet fully elucidated.

\subsubsection{Coronavirus Disease 19 (COVID-19)}

COVID-19 is a respiratory illness initially identified as an unfamiliar pneumonia in a group of patients in December 2019 in Wuhan, China, initially named as 2019 novel coronavirus by the World Health Organization (WHO) on January 7 of 2020 [196]. It is caused by a new coronavirus, which due to $86.9 \%$ genome similarity with the Severe Acute Respiratory Syndrome Coronavirus (SARS-CoV) was named SARS-CoV-2. COVID-19 presents with influenza-like symptoms ranging from mild disease to severe lung injury, multi-organ failure and eventually leading to death, especially in older patients or patients with other comorbidities [196-198]. SARS-CoV-2 infects the host cells through the angiotensin converting enzyme 2 (ACE2) receptor, which is also expressed in endothelial cells. Thus, in addition to respiratory disease, SARS-CoV-2 virus has been shown to infect blood vessels in multiple organs and induce vascular damage in vitro and endothelial inflammation (endotheliitis) in patients $[197,199,200]$. Vascular dysfunction is an underlying feature of the pathophysiology of most respiratory conditions, including COVID-19 and ARDS, especially in patients with pre-existing comorbidities [200, 201]. Some studies have demonstrated that SARS-CoV-2 infects pulmonary endothelial cells and causes microvascular leaks, contributing to the initiation and propagation of respiratory distress and ARDS in COVID-19 patients by altering blood vessel barrier integrity, among other parameters [202].

A few studies have implicated Ang2 with the severity and outcome of COVID-19. The levels of Ang2 in the plasma were positively associated with the viral RNA load in plasma of COVID-19 patients, demonstrating a potential role of Ang2 in the endothelial dysfunction severity, a pivotal feature in COVID-19 [203]. Ang2 was one of the two markers, along with E-selectin, detected upregulated in plasma of COVID-19 patients, and was reported as a predictive factor for intensive care unit (ICU) admission, with a cut-off of $5000 \mathrm{pg} / \mathrm{mL}$ as the best predictor for ICU outcome [200]. Plasma Ang2 levels were also associated with in-hospital mortality and non-resolving pulmonary condition [204]. The above indicate the involvement of Ang2 in endothelial activation and highlight it as an important player in the pathophysiology of COVID-19-associated microvascular dysfunction.

\subsection{Drugs Targeting the Ang/Tie Pathway in Pulmonary Vascular Disorders}

Despite the obvious role of members of the Ang/Tie family in the etiology and prognosis of respiratory conditions, so far, there is limited focus on their role in the clinical setting. In the case of the pulmonary vascular disorders, the sole targets for the investigational new drugs are Ang2 and Tie2, demonstrating that the lack of molecules targeting other important members of the Ang/Tie family is an area worth exploring. Below, we report the targeted clinical approaches, classified according to the target molecules, currently in clinical trials that target the Ang/Tie signaling pathway for pulmonary vascular disorders (Fig. 1).

\subsubsection{Tie2 Receptor}

Vasculotide: Vasculotide (Vasomune Therapeutics) is a Tie2-agonist tetrameric peptide that improves survival in murine models of severe influenza. The aim of this molecule was to tetramerically bind and cluster Tie 2 receptors in an 'Ang1-like' manner to activate Tie2 signaling inducing Ang1-like cellular and physiological responses [205]. While the effect of vasculotide was not related to viral replication, nor did it affect neutrophil recruitment, it decreased lung edema, hypoxemia, and lung endothelial apoptosis [205]. Vascular leakiness is one of the common features in 
pulmonary vascular disorders and sepsis. Vasculotide, had been previously evaluated in a sepsis model, where it prevented capillary leakiness, neutrophil transmigration, and overall rescued mice from sepsis-related death [206]. Similarly, vasculotide prevented endotoxin-induced lung vascular leakage via Tie 2 and downstream Akt activation in an endotoxemic mouse lung injury model. Pre- and post-treatment with vasculotide improved survival, an effect that was lost in Tie2 heterozygosity, demonstrating its specificity to Tie2 receptor [207]. In these studies, vasculotide was shown to act through binding and phosphorylation of Tie2, findings in agreement with Tournaire et al, who were to identify T7 (later modified into vasculotide [208]), showing that it binds with high affinity to the extracellular portion of the Tie2 receptor but lacks the capacity to displace either Ang1 or Ang2 [209]. Two mechanisms have been pinpointed for vasculotide's effects: the increased activation of Tie2 and the inhibition of the proinflammatory and chemotactic cytokine release [206]. A more recent study excluded the first mechanism, as vasculotide applied as a potential therapy in tumor cell extravasation, was reported not to bind to the extracellular domain of Tie2, as previously stated by other researchers. Furthermore, it did not induce Tie 2 phosphorylation, nor act as a direct agonist of the Tie 2 receptor [210]. The above studies agree with the effect of vasculotide on stabilizing the lung vasculature, but conflict on the mechanism of action. The effect of vasculotide on molecules regulating Tie2 phosphorylation and endothelium stabilization such as Ang2, VE-PTP, Tie1 and integrins needs to be investigated. As previously mentioned, T7 did not compete with Ang1 or Ang2 for binding to Tie2 [209], therefore more evaluation is required for delineating the interaction with Tie2. High concentrations of clustered vasculotide did not activate Tie2 [208], which could also be a possible explanation for the noted differences in these studies. Vasculotide, was the predecessor of $\mathrm{AV}-001$, analyzed below.

AV-001: AV-001 (Vasomune Therapeutics) is a novel investigational drug that targets the Tie 2 receptor, which is highly expressed on the surface of endothelial cells in the vasculature of the lungs, among other organs. AV-001 acts by activating the Tie2-angiopoietin pathway restoring normal vascular function and endothelial stability [211]. The same company has reported a previous study, where vasculotide, a Tie2-agonist tetrameric peptide, improves survival in murine models of severe influenza [205]. During the preclinical studies of the AV-001, in which a lethal RNA virus infection animal model of influenza/ARDS was used, AV-001 stabilized the vasculature by enhancing endothelial cell stability, blocking vascular leakiness, and restoring normal endothelial barrier function. As a monotherapy, AV-001 improved survival and lung function with an enhanced recovery in combination with antiviral therapy. AV-001 is currently in a Phase 1 double-blind, placebo-controlled single and multiple ascending dose study with COVID-19, ARDS or COVID-19-associated ARDS patients for safety and tolerability assessments. Should the Phase 1 results show AV-001 to be safe and well-tolerated, Phase 2 will be initiated as a proof-of-concept study to assess efficacy in patients with moderate-to-severe COVID-19 disease [211, 212]. Based on the knowledge from the trials in retinal disorders, combination of AV-001 with anti-VEGF inhibitors could provide optimal results and would be worth exploring, depending on the success of the Phase 1 trials.

\subsubsection{Angiopoietin 2 (Ang2)}

LY3127804: LY3127804 (Eli Lilly) is a humanized antibody that recognizes and binds Ang2 with high affinity, blocking Ang2 activity. After the successful outcome of combinatorial treatments of LY3127804 with D101, a murine antiVEGFR2 antibody in mouse tumor models, the combination of LY3127804 with ramucirumab, a monoclonal antibody targeting VEGFR2, was well tolerated in a Phase 1 clinical trial, indicating clinical activity [213]. A randomized, double-blind, placebo-controlled clinical trial of LY3127804 in pneumonia patients with presumed or confirmed COVID19 was designed to identify the effect of Ang2 inhibition in COVID-19-related ARDS. The study has been completed with the results being expected soon [214].

ABTAA: ABTAA is the Ang2-binding and Tie2-activating antibody mentioned earlier to be effective for NV-AMD. The potency of ABTAA was equally promising in sepsis, as it prevented septic damage in the vasculature and protected patients from sepsis-related death in three sepsis models. As part of Tie2 activation, it prevented vascular leakage, strengthened endothelial glycocalyx and reduced cytokine storm [147].

Ang2 targeting for respiratory conditions is justified, given its reported role in pulmonary disorders, such as COVID-19, where Ang2 was identified as a critical determinant for ICU admission and severity. However, the absence of a considerable number of successful clinical trials, inadequate knowledge on potential tissue-specific differences on endothelial physiology and alterations of the mechanisms, as well as overlap with other pathways, are factors that do not allow safe prediction of the outcome of Ang2 targeting for pulmonary disorders.

\section{Conclusions}

The accumulating data regarding the role of the Ang/Tie signaling pathway in endothelial physiology, and the impact this has in vascular diseases has revealed the significance of targeting this pathway in vascular-related diseases of certain 
organs, among which are the retina and the lung. The role of the Ang/Tie signaling pathway in determining the status of the vascular endothelium is evident and its function in retinal and respiratory vasculature, especially in disease states, is sufficiently known to support the growing number of clinical trials. Although the focus on the members of the Ang/Tie family as potential targets, especially in pulmonary diseases, is still limited; side effects of current treatments, as well as the limited therapeutic options, increase the need to pursue new therapeutic targets, with angiopoietins and Tie receptors being at the top of the list. However, although most of the clinical trials have yielded positive outcome, the comparison with the current treatment option is modest in most cases. The drug candidates targeting the Ang/Tie pathway, regardless of the primary target (Tie2, VE-PTP or Ang2) all aim at inducing Tie 2 signaling. The basic research has highlighted the significance of this pathway and is supportive of targeting the Ang/Tie pathway for vascular and lymphatic vascular disorders. Modifications in the formulation and the routes of administration of the drug candidates could improve the outcome, given that the number of Ang/Tietargeting drug candidates and clinical trials are increasing. Moreover, the fact that members of this pathway, such as Ang2 are not present or elevated in normal conditions provides specificity of targets, minimizing side effects. Based, on current data, combination therapy for the Ang/Tie and the VEGF pathways should yield improved clinical outcome and would minimize the chances for recurrence and compensatory mechanisms. At the same time, basic research focusing on the interplay among Ant/Tie members themselves or with other vascular mediators will open up the potential for treatments with higher effectiveness and more permanent outcomes. It is expected that more drug candidates for the Ang/Tie pathway will appear in the future and the focus towards this pathway as a clinical target for monotherapy or adjunct therapy will increase, providing solutions on retinal and the less studied pulmonary vascular disorders.

\section{Declarations}

Funding This work was supported in part by the National Institutes of Health Grant (NCI) R15CA231339 and the Texas Tech University Health Sciences Center (TTUHSC) School of Pharmacy Office of the Sciences grant. The funders had no role in study design, decision to write, or preparation of the manuscript.

Conflict of interest The authors declare no competing interests.

Ethics approval Not applicable.

Consent Not applicable.

Author contributions Conceptualization, RGA and CMM; writingreview and editing, RGA, CMM; funding acquisition: CMM.
Data availability statement Not applicable.

\section{References}

1. Dumont DJ, et al. The endothelial-specific receptor tyrosine kinase, tek, is a member of a new subfamily of receptors. Oncogene. 1993;8(5):1293-301.

2. Sato TN, et al. Distinct roles of the receptor tyrosine kinases Tie-1 and Tie-2 in blood vessel formation. Nature. 1995;376(6535):70-4.

3. Maisonpierre PC, et al. Angiopoietin-2, a natural antagonist for Tie 2 that disrupts in vivo angiogenesis. Science. 1997;277(5322):55-60.

4. Jones $\mathrm{N}$, et al. Identification of Tek/Tie2 binding partners. Binding to a multifunctional docking site mediates cell survival and migration. J Biol Chem. 1999;274(43):30896-905.

5. Akwii RG, et al. Role of angiopoietin-2 in vascular physiology and pathophysiology. Cells. 2019;8(5):471.

6. Kim I, et al. Angiopoietin-2 at high concentration can enhance endothelial cell survival through the phosphatidylinositol 3'-kinase/Akt signal transduction pathway. Oncogene. 2000;19(39):4549-52.

7. Nguyen VP, et al. Differential response of lymphatic, venous and arterial endothelial cells to angiopoietin-1 and angiopoietin-2. BMC Cell Biol. 2007;8:10.

8. Andrawes NG, et al. Angiopoietin-2 as a marker of retinopathy in children and adolescents with sickle cell disease: relation to subclinical atherosclerosis. J Pediatr Hematol Oncol. 2019;41(5):361-70.

9. Kinnen A, et al. Gene expression in the Angiopoietin/TIE axis is altered in peripheral tissue of ovarian cancer patients: a prospective observational study. Life Sci. 2021;274:119345.

10. Pirouzpanah S, et al. The contribution of dietary and plasma folate and cobalamin to levels of angiopoietin-1, angiopoietin-2 and Tie-2 receptors depend on vascular endothelial growth factor status of primary breast cancer patients. Sci Rep. 2019;9(1):14851.

11. van der Heijden M, et al. Angiopoietin-2, permeability oedema, occurrence and severity of ALI/ARDS in septic and non-septic critically ill patients. Thorax. 2008;63(10):903-9.

12. Parikh SM, et al. Excess circulating angiopoietin-2 may contribute to pulmonary vascular leak in sepsis in humans. PLoS Med. 2006;3(3):e46.

13. Kim I, et al. Angiopoietin-1 induces endothelial cell sprouting through the activation of focal adhesion kinase and plasmin secretion. Circ Res. 2000;86(9):952-9.

14. Papapetropoulos A, et al. Direct actions of angiopoietin-1 on human endothelium: evidence for network stabilization, cell survival, and interaction with other angiogenic growth factors. Lab Invest. 1999;79(2):213-23.

15. Davis $\mathrm{S}$, et al. Isolation of angiopoietin-1, a ligand for the TIE2 receptor, by secretion-trap expression cloning. Cell. 1996;87(7):1161-9.

16. Dumont DJ, et al. Dominant-negative and targeted null mutations in the endothelial receptor tyrosine kinase, tek, reveal a critical role in vasculogenesis of the embryo. Genes Dev. 1994;8(16):1897-909.

17. Chu M, et al. Angiopoietin receptor Tie2 is required for vein specification and maintenance via regulating COUP-TFII. Elife. 2016;5:e21032.

18. Patan S. TIE1 and TIE2 receptor tyrosine kinases inversely regulate embryonic angiogenesis by the mechanism of intussusceptive microvascular growth. Microvasc Res. 1998;56(1):1-21. 
19. Suri C, et al. Requisite role of angiopoietin-1, a ligand for the TIE2 receptor, during embryonic angiogenesis. Cell. 1996;87(7):1171-80.

20. Hansen TM, et al. Effects of angiopoietins-1 and -2 on the receptor tyrosine kinase Tie 2 are differentially regulated at the endothelial cell surface. Cell Signal. 2010;22(3):527-32.

21. Kim I, et al. Angiopoietin-1 regulates endothelial cell survival through the phosphatidylinositol 3'-Kinase/Akt signal transduction pathway. Circ Res. 2000;86(1):24-9.

22. Harfouche R, et al. Mechanisms which mediate the antiapoptotic effects of angiopoietin-1 on endothelial cells. Microvasc Res. 2002;64(1):135-47.

23. Kim YM, et al. Hydrogen peroxide produced by angiopoietin-1 mediates angiogenesis. Cancer Res. 2006;66(12):6167-74.

24. Sako K, et al. Angiopoietin-1 induces Kruppel-like factor 2 expression through a phosphoinositide 3-kinase/AKT-dependent activation of myocyte enhancer factor 2. J Biol Chem. 2009;284(9):5592-601.

25. Zhang J, et al. Angiopoietin-1/Tie2 signal augments basal Notch signal controlling vascular quiescence by inducing delta-like 4 expression through AKT-mediated activation of beta-catenin. J Biol Chem. 2011;286(10):8055-66.

26. Gavard J, Patel V, Gutkind JS. Angiopoietin-1 prevents VEGFinduced endothelial permeability by sequestering Src through mDia. Dev Cell. 2008;14(1):25-36.

27. Oubaha M, Gratton JP. Phosphorylation of endothelial nitric oxide synthase by atypical PKC zeta contributes to angiopoietin-1-dependent inhibition of VEGF-induced endothelial permeability in vitro. Blood. 2009;114(15):3343-51.

28. Gao F, et al. Modulation of long-term endothelial-barrier integrity is conditional to the cross-talk between Akt and Src signaling. J Cell Physiol. 2017;232(10):2599-609.

29. Teichert-Kuliszewska K, et al. Biological action of angiopoietin-2 in a fibrin matrix model of angiogenesis is associated with activation of Tie2. Cardiovasc Res. 2001;49(3):659-70.

30. Yuan HT, et al. Angiopoietin 2 is a partial agonist/antagonist of Tie2 signaling in the endothelium. Mol Cell Biol. 2009;29(8):2011-22.

31. Eklund L, Saharinen P. Angiopoietin signaling in the vasculature. Exp Cell Res. 2013;319(9):1271-80.

32. Holash J, et al. Vessel cooption, regression, and growth in tumors mediated by angiopoietins and VEGF. Science. 1999;284(5422):1994-8.

33. Fiedler $\mathrm{U}$, et al. The Tie- 2 ligand angiopoietin- 2 is stored in and rapidly released upon stimulation from endothelial cell Weibel-Palade bodies. Blood. 2004;103(11):4150-6.

34. Kim M, et al. Opposing actions of angiopoietin-2 on Tie2 signaling and FOXO1 activation. J Clin Invest. 2016;126(9):3511-25.

35. Korhonen EA, et al. Tie 1 controls angiopoietin function in vascular remodeling and inflammation. J Clin Invest. 2016;126(9):3495-510.

36. Song $\mathrm{SH}$, et al. Tie 1 regulates the Tie 2 agonistic role of angiopoietin-2 in human lymphatic endothelial cells. Biochem Biophys Res Commun. 2012;419(2):281-6.

37. Singh $\mathrm{H}$, et al. Vascular endothelial growth factor activates the Tie family of receptor tyrosine kinases. Cell Signal. 2009;21(8):1346-50.

38. Gale NW, et al. Angiopoietin-2 is required for postnatal angiogenesis and lymphatic patterning, and only the latter role is rescued by Angiopoietin-1. Dev Cell. 2002;3(3):411-23.

39. Singh $\mathrm{H}$, et al. The molecular balance between receptor tyrosine kinases Tie 1 and Tie 2 is dynamically controlled by VEGF and TNFalpha and regulates angiopoietin signalling. PLoS ONE. 2012;7(1):e29319.
40. Leppanen VM, Saharinen P, Alitalo K. Structural basis of Tie2 activation and Tie2/Tie1 heterodimerization. Proc Natl Acad Sci USA. 2017;114(17):4376-81.

41. Yabkowitz R, et al. Inflammatory cytokines and vascular endothelial growth factor stimulate the release of soluble tie receptor from human endothelial cells via metalloprotease activation. Blood. 1999;93(6):1969-79.

42. Yabkowitz R, et al. Regulation of tie receptor expression on human endothelial cells by protein kinase $\mathrm{C}$-mediated release of soluble tie. Blood. 1997;90(2):706-15.

43. McCarthy MJ, et al. Potential roles of metalloprotease mediated ectodomain cleavage in signaling by the endothelial receptor tyrosine kinase Tie-1. Lab Invest. 1999;79(7):889-95.

44. Chen-Konak L, et al. Transcriptional and post-translation regulation of the Tie1 receptor by fluid shear stress changes in vascular endothelial cells. FASEB J. 2003;17(14):2121-3.

45. Marron MB, et al. Evidence for heterotypic interaction between the receptor tyrosine kinases TIE-1 and TIE-2. J Biol Chem. 2000;275(50):39741-6.

46. Marron MB, et al. Tie-1 receptor tyrosine kinase endodomain interaction with SHP2: potential signalling mechanisms and roles in angiogenesis. Adv Exp Med Biol. 2000;476:35-46.

47. Findley CM, et al. VEGF induces Tie2 shedding via a phosphoinositide 3-kinase/Akt dependent pathway to modulate Tie2 signaling. Arterioscler Thromb Vasc Biol. 2007;27(12):2619-26.

48. Reusch P, et al. Identification of a soluble form of the angiopoietin receptor TIE-2 released from endothelial cells and present in human blood. Angiogenesis. 2001;4(2):123-31.

49. Onimaru M, et al. An autocrine linkage between matrix metalloproteinase-14 and Tie-2 via ectodomain shedding modulates angiopoietin-1-dependent function in endothelial cells. Arterioscler Thromb Vasc Biol. 2010;30(4):818-26.

50. Goel S, et al. Effects of vascular-endothelial protein tyrosine phosphatase inhibition on breast cancer vasculature and metastatic progression. J Natl Cancer Inst. 2013;105(16):1188-201.

51. Fachinger G, Deutsch U, Risau W. Functional interaction of vascular endothelial-protein-tyrosine phosphatase with the angiopoietin receptor Tie-2. Oncogene. 1999;18(43):5948-53.

52. Saharinen $P$, et al. Angiopoietins assemble distinct Tie2 signalling complexes in endothelial cell-cell and cell-matrix contacts. Nat Cell Biol. 2008;10(5):527-37.

53. Winderlich M, et al. VE-PTP controls blood vessel development by balancing Tie-2 activity. J Cell Biol. 2009;185(4):657-71.

54. Nawroth R, et al. VE-PTP and VE-cadherin ectodomains interact to facilitate regulation of phosphorylation and cell contacts. EMBO J. 2002;21(18):4885-95.

55. Nottebaum AF, et al. VE-PTP maintains the endothelial barrier via plakoglobin and becomes dissociated from VE-cadherin by leukocytes and by VEGF. J Exp Med. 2008;205(12):2929-45.

56. Bäumer $\mathrm{S}$, et al. Vascular endothelial cell-specific phosphotyrosine phosphatase (VE-PTP) activity is required for blood vessel development. Blood. 2006;107(12):4754-62.

57. Dominguez MG, et al. Vascular endothelial tyrosine phosphatase (VE-PTP)-null mice undergo vasculogenesis but die embryonically because of defects in angiogenesis. Proc Natl Acad Sci USA. 2007;104(9):3243-8.

58. Frye M, et al. Interfering with VE-PTP stabilizes endothelial junctions in vivo via Tie-2 in the absence of VE-cadherin. J Exp Med. 2015;212(13):2267-87.

59. Broermann A, et al. Dissociation of VE-PTP from VE-cadherin is required for leukocyte extravasation and for VEGF-induced vascular permeability in vivo. J Exp Med. 2011;208(12):2393-401.

60. Fiedler U, et al. Angiopoietin-2 sensitizes endothelial cells to TNF-alpha and has a crucial role in the induction of inflammation. Nat Med. 2006;12(2):235-9. 
61. Buehler D, et al. Expression of angiopoietin-TIE system components in angiosarcoma. Mod Pathol. 2013;26(8):1032-40.

62. Hasenstein JR, et al. Efficacy of Tie2 receptor antagonism in angiosarcoma. Neoplasia. 2012;14(2):131-40.

63. Wong-Riley MT. Energy metabolism of the visual system. Eye Brain. 2010;2:99-116.

64. Hayreh SS. The cilio-retinal arteries. Br J Ophthalmol. 1963;47:71-89.

65. Pournaras CJ, et al. Regulation of retinal blood flow in health and disease. Prog Retin Eye Res. 2008;27(3):284-330.

66. Saint-Geniez M, D'Amore PA. Development and pathology of the hyaloid, choroidal and retinal vasculature. Int J Dev Biol. 2004;48(8-9):1045-58.

67. Dreher Z, Robinson SR, Distler C. Muller cells in vascular and avascular retinae: a survey of seven mammals. J Comp Neurol. 1992;323(1):59-80.

68. Potente M, Gerhardt H, Carmeliet P. Basic and therapeutic aspects of angiogenesis. Cell. 2011;146(6):873-87.

69. Al-Latayfeh M, et al. Antiangiogenic therapy for ischemic retinopathies. Cold Spring Harb Perspect Med. 2012;2(6):a006411.

70. Sharif Z, Sharif W. Corneal neovascularization: updates on pathophysiology, investigations \& management. Rom J Ophthalmol. 2019;63(1):15-22.

71. Hackett SF, et al. Angiopoietin-2 plays an important role in retinal angiogenesis. J Cell Physiol. 2002;192(2):182-7.

72. Hackett SF, et al. Angiopoietin 2 expression in the retina: upregulation during physiologic and pathologic neovascularization. J Cell Physiol. 2000;184(3):275-84.

73. Gengenbacher N, et al. Timed Ang2-targeted therapy identifies the angiopoietin-tie pathway as key regulator of fatal lymphogenous metastasis. Cancer Discov. 2021;11(2):424-45.

74. Kapiainen E, et al. The amino-terminal oligomerization domain of Angiopoietin-2 affects vascular remodeling, mammary gland tumor growth, and lung metastasis in mice. Cancer Res. 2021;81(1):129-143.

75. Nambu H, et al. Angiopoietin 1 prevents retinal detachment in an aggressive model of proliferative retinopathy, but has no effect on established neovascularization. J Cell Physiol. 2005;204(1):227-35.

76. Dumont DJ, et al. Vascularization of the mouse embryo: a study of flk-1, tek, tie, and vascular endothelial growth factor expression during development. Dev Dyn. 1995;203(1):80-92.

77. Fruttiger M. Development of the retinal vasculature. Angiogenesis. 2007;10(2):77-88.

78. Selvam S, Kumar T, Fruttiger M. Retinal vasculature development in health and disease. Prog Retin Eye Res. 2018;63:1-19.

79. Tuerk C, Gold L. Systematic evolution of ligands by exponential enrichment: RNA ligands to bacteriophage T4 DNA polymerase. Science. 1990;249(4968):505-10.

80. Ng EW, et al. Pegaptanib, a targeted anti-VEGF aptamer for ocular vascular disease. Nat Rev Drug Discov. 2006;5(2):123-32.

81. Michels S, et al. Systemic bevacizumab (Avastin) therapy for neovascular age-related macular degeneration twelve-week results of an uncontrolled open-label clinical study. Ophthalmology. 2005;112(6):1035-47.

82. Rosenfeld PJ, Moshfeghi AA, Puliafito CA. Optical coherence tomography findings after an intravitreal injection of bevacizumab (avastin) for neovascular age-related macular degeneration. Ophthalmic Surg Lasers Imaging. 2005;36(4):331-5.

83. Rosenfeld PJ, Fung AE, Puliafito CA. Optical coherence tomography findings after an intravitreal injection of bevacizumab (avastin) for macular edema from central retinal vein occlusion. Ophthalmic Surg Lasers Imaging. 2005;36(4):336-9.

84. Chen Y, et al. Selection and analysis of an optimized anti-VEGF antibody: crystal structure of an affinity-matured Fab in complex with antigen. J Mol Biol. 1999;293(4):865-81.
85. Ferrara N, et al. Development of ranibizumab, an anti-vascular endothelial growth factor antigen binding fragment, as therapy for neovascular age-related macular degeneration. Retina. 2006;26(8):859-70.

86. Rosenfeld PJ, et al. Ranibizumab for neovascular age-related macular degeneration. N Engl J Med. 2006;355(14):1419-31.

87. Brown DM, et al. Ranibizumab versus verteporfin for neovascular age-related macular degeneration. N Engl J Med. 2006;355(14):1432-44.

88. Kim H, Robinson SB, Csaky KG. FcRn receptor-mediated pharmacokinetics of therapeutic IgG in the eye. Mol Vis. 2009;15:2803-12.

89. Heiduschka P, et al. Penetration of bevacizumab through the retina after intravitreal injection in the monkey. Invest Ophthalmol Vis Sci. 2007;48(6):2814-23.

90. Gerber HP, et al. VEGF couples hypertrophic cartilage remodeling, ossification and angiogenesis during endochondral bone formation. Nat Med. 1999;5(6):623-8.

91. Ferrara N, et al. Vascular endothelial growth factor is essential for corpus luteum angiogenesis. Nat Med. 1998;4(3):336-40.

92. Holash J, et al. VEGF-Trap: a VEGF blocker with potent antitumor effects. Proc Natl Acad Sci USA. 2002;99(17):11393-8.

93. Yun JH, et al. Angiopoietin 2 induces astrocyte apoptosis via av $\beta 5$-integrin signaling in diabetic retinopathy. Cell Death Dis. 2016;7(2):e2101.

94. Cai J, et al. The angiopoietin/Tie-2 system regulates pericyte survival and recruitment in diabetic retinopathy. Invest Ophthalmol Vis Sci. 2008;49(5):2163-71.

95. Soto I, et al. Vascular inflammation risk factors in retinal disease. Annu Rev Vis Sci. 2019;5:99-122.

96. Wong TY, Mitchell P. The eye in hypertension. Lancet. 2007;369(9559):425-35.

97. Le HG, Shakoor A. Diabetic and retinal vascular eye disease. Med Clin N Am. 2021;105(3):455-72.

98. DellaCroce JT, Vitale AT. Hypertension and the eye. Curr Opin Ophthalmol. 2008;19(6):493-8.

99. Bhargava M, Ikram MK, Wong TY. How does hypertension affect your eyes? J Hum Hypertens. 2012;26(2):71-83.

100. Brand CS. Management of retinal vascular diseases: a patientcentric approach. Eye (Lond). 2012;26(Suppl 2):S1-16.

101. Al-Zamil WM, Yassin SA. Recent developments in agerelated macular degeneration: a review. Clin Interv Aging. 2017;12:1313-30.

102. Mitchell P, et al. Age-related macular degeneration. Lancet. 2018;392(10153):1147-59.

103. Bhutto I, Lutty G. Understanding age-related macular degeneration (AMD): relationships between the photoreceptor/retinal pigment epithelium/Bruch's membrane/choriocapillaris complex. Mol Aspects Med. 2012;33(4):295-317.

104. Kim J, et al. Tie2 activation promotes choriocapillary regeneration for alleviating neovascular age-related macular degeneration. Sci Adv. 2019;5(2):eaau6732.

105. Maguire MG, et al. Five-year outcomes with anti-vascular endothelial growth factor treatment of neovascular agerelated macular degeneration: the comparison of age-related macular degeneration treatments trials. Ophthalmology. 2016;123(8):1751-61.

106. Young M, et al. Exacerbation of choroidal and retinal pigment epithelial atrophy after anti-vascular endothelial growth factor treatment in neovascular age-related macular degeneration. Retina. 2014;34(7):1308-15.

107. Kurihara T, et al. Targeted deletion of Vegfa in adult mice induces vision loss. J Clin Invest. 2012;122(11):4213-7.

108. Saint-Geniez M, et al. An essential role for RPE-derived soluble VEGF in the maintenance of the choriocapillaris. Proc Natl Acad Sci USA. 2009;106(44):18751-6. 
109. Sharma A, et al. Faricimab: expanding horizon beyond VEGF. Eye (Lond). 2020;34(5):802-4.

110. Regula JT, et al. Targeting key angiogenic pathways with a bispecific CrossMAb optimized for neovascular eye diseases. EMBO Mol Med. 2016;8(11):1265-88.

111. $\mathrm{Ng}$ DS, et al. Elevated angiopoietin 2 in aqueous of patients with neovascular age related macular degeneration correlates with disease severity at presentation. Sci Rep. 2017;7:45081.

112. Shen J, et al. Targeting VE-PTP activates TIE2 and stabilizes the ocular vasculature. J Clin Invest. 2014;124(10):4564-76.

113. Nambu H, et al. Angiopoietin 1 inhibits ocular neovascularization and breakdown of the blood-retinal barrier. Gene Ther. 2004;11(10):865-73.

114. Wang W, Lo ACY. Diabetic retinopathy: pathophysiology and treatments. Int J Mol Sci. 2018;19(6):1816.

115. Romero-Aroca P, et al. Diabetic macular edema pathophysiology: vasogenic versus inflammatory. J Diabetes Res. 2016;2016:2156273.

116. Das A, McGuire PG, Rangasamy S. Diabetic macular edema: pathophysiology and novel therapeutic targets. Ophthalmology. 2015;122(7):1375-94.

117. Daruich A, et al. Mechanisms of macular edema: beyond the surface. Prog Retin Eye Res. 2018;63:20-68.

118. Arjamaa O, Nikinmaa M. Oxygen-dependent diseases in the retina: role of hypoxia-inducible factors. Exp Eye Res. 2006;83(3):473-83.

119. Urias EA, et al. Novel therapeutic targets in diabetic macular edema: beyond VEGF. Vis Res. 2017;139:221-7.

120. Adamis AP, Berman AJ. Immunological mechanisms in the pathogenesis of diabetic retinopathy. Semin Immunopathol. 2008;30(2):65-84.

121. Elman MJ, et al. Randomized trial evaluating ranibizumab plus prompt or deferred laser or triamcinolone plus prompt laser for diabetic macular edema. Ophthalmology. 2010;117(6):10641077.e35.

122. Sang DN, D'Amore PA. Is blockade of vascular endothelial growth factor beneficial for all types of diabetic retinopathy? Diabetologia. 2008;51(9):1570-3.

123. David S, et al. Angiopoietin-2 may contribute to multiple organ dysfunction and death in sepsis*. Crit Care Med. 2012;40(11):3034-41.

124. Ando M, et al. Angiopoietin-2 expression in patients with an acute exacerbation of idiopathic interstitial pneumonias. Respir Med. 2016;117:27-32.

125. Clajus $\mathrm{C}$, et al. Angiopoietin-2 is a potential mediator of endothelial barrier dysfunction following cardiopulmonary bypass. Cytokine. 2012;60(2):352-9.

126. Rangasamy S, McGuire PG, Das A. Diabetic retinopathy and inflammation: novel therapeutic targets. Middle East Afr J Ophthalmol. 2012;19(1):52-9.

127. Eklund L, Kangas J, Saharinen P. Angiopoietin-Tie signalling in the cardiovascular and lymphatic systems. Clin Sci (Lond). 2017;131(1):87-103.

128. Aspelund A, et al. The Schlemm's canal is a VEGF-C/ VEGFR-3-responsive lymphatic-like vessel. J Clin Invest. 2014;124(9):3975-86.

129. Park DY, et al. Lymphatic regulator PROX1 determines Schlemm's canal integrity and identity. J Clin Invest. 2014;124(9):3960-74.

130. QBioMed. 2021. https://qbiomed.com/pipeline/man-01.

131. Insight, A. MAN 01. 2020. https://adisinsight.springer.com/ drugs/800058669.

132. Campochiaro PA, et al. Enhanced benefit in diabetic macular edema from AKB-9778 Tie2 activation combined with vascular endothelial growth factor suppression. Ophthalmology. 2016;123(8):1722-30.
133. Pharmaceuticals, A. Razuprotafib (AKB-9778) diabetic nephropathy. 2020. https://aerpio.com/pipeline/razuprotafib-akb-9778diabetic-nephropathy/.

134. Hussain RM, et al. Tie-2/Angiopoietin pathway modulation as a therapeutic strategy for retinal disease. Expert Opin Investig Drugs. 2019;28(10):861-9.

135. Pharmaceuticals, A. ARP-1536 Retinopathy/Nephropathy. 2020. https://aerpio.com/pipeline/arp-1536-diabetic-retinopathy-nephr opathy/.

136. Klein C, Schaefer W, Regula JT. The use of CrossMAb technology for the generation of bi- and multispecific antibodies. MAbs. 2016;8(6):1010-20.

137. Roche H-L. ClinialTrials.gov-Faricimab. 2020. https://clinicaltr ials.gov/ct $2 /$ results?cond $=\&$ term $=$ faricimab $\&$ cntry $=\&$ state $=\&$ city $=\&$ dist $=$

138. Chong V. Ranibizumab for the treatment of wet AMD: a summary of real-world studies. Eye (Lond). 2016;30(2):270-86.

139. Blick SK, Keating GM, Wagstaff AJ. Ranibizumab. Drugs. 2007;67(8):1199-206 (discussion 1207-1209).

140. Sahni J, et al. Safety and efficacy of different doses and regimens of faricimab vs ranibizumab in neovascular age-related macular degeneration: the AVENUE phase 2 randomized clinical trial. JAMA Ophthalmol. 2020;138(9):955-63.

141. Sahni J, et al. Simultaneous inhibition of angiopoietin-2 and vascular endothelial growth factor-A with faricimab in diabetic macular edema: BOULEVARD phase 2 randomized trial. Ophthalmology. 2019;126(8):1155-70.

142. Khanani AM, et al. Efficacy of every four monthly and quarterly dosing of faricimab vs ranibizumab in neovascular age-related macular degeneration: the STAIRWAY phase 2 randomized clinical trial. JAMA Ophthalmol. 2020;138(9):964-72.

143. Lee JY, et al. Regulation of angiopoietin-2 secretion from human pulmonary microvascular endothelial cells. Exp Lung Res. 2016;42(7):335-45.

144. Huang YQ, et al. Thrombin induces increased expression and secretion of angiopoietin-2 from human umbilical vein endothelial cells. Blood. 2002;99(5):1646-50.

145. Papadopoulos KP, et al. A Phase I first-in-human study of nesvacumab (REGN910), a fully human anti-angiopoietin-2 (Ang2) monoclonal antibody, in patients with advanced solid tumors. Clin Cancer Res. 2016;22(6):1348-55.

146. Kim J, et al. Impaired angiopoietin/Tie2 signaling compromises Schlemm's canal integrity and induces glaucoma. J Clin Invest. 2017;127(10):3877-96.

147. Han S, et al. Amelioration of sepsis by TIE2 activation-induced vascular protection. Sci Transl Med. 2016;8(335):335ra55.

148. Souma T, et al. Context-dependent functions of angiopoietin 2 are determined by the endothelial phosphatase VEPTP. Proc Natl Acad Sci USA. 2018;115(6):1298-303.

149. Takagi $\mathrm{H}$, et al. Potential role of the angiopoietin/tie 2 system in ischemia-induced retinal neovascularization. Invest Ophthalmol Vis Sci. 2003;44(1):393-402.

150. Li W, et al. Soluble Tei2 fusion protein inhibits retinopathy of prematurity occurrence via regulation of the Ang/Tie2 pathway. Exp Ther Med. 2019;18(1):614-20.

151. Gao Y, Raj JU. Regulation of the pulmonary circulation in the fetus and newborn. Physiol Rev. 2010;90(4):1291-335.

152. Grzenda A, et al. Timing and expression of the angiopoietin1-Tie-2 pathway in murine lung development and congenital diaphragmatic hernia. Dis Model Mech. 2013;6(1):106-14.

153. Healy AM, et al. VEGF is deposited in the subepithelial matrix at the leading edge of branching airways and stimulates neovascularization in the murine embryonic lung. Dev Dyn. 2000;219(3):341-52.

154. Tirziu D, Simons M. Endothelium as master regulator of organ development and growth. Vascul Pharmacol. 2009;50(1-2):1-7. 
155. Dong Z, et al. Ang-2 promotes lung cancer metastasis by increasing epithelial-mesenchymal transition. Oncotarget. 2018;9(16):12705-17.

156. $\mathrm{Xu} \mathrm{Y,} \mathrm{et} \mathrm{al.} \mathrm{The} \mathrm{role} \mathrm{of} \mathrm{serum} \mathrm{angiopoietin-2} \mathrm{levels} \mathrm{in} \mathrm{progres-}$ sion and prognosis of lung cancer: a meta-analysis. Medicine (Baltimore). 2017;96(37):e8063.

157. Reilly JP, et al. Plasma angiopoietin-2 as a potential causal marker in sepsis-associated ARDS development: evidence from Mendelian randomization and mediation analysis. Intensive Care Med. 2018;44(11):1849-58.

158. Matthay MA, Ware LB, Zimmerman GA. The acute respiratory distress syndrome. J Clin Invest. 2012;122(8):2731-40.

159. Calfee CS, et al. Plasma angiopoietin-2 in clinical acute lung injury: prognostic and pathogenetic significance. Crit Care Med. 2012;40(6):1731-7.

160. Bhandari V, et al. Hyperoxia causes angiopoietin 2-mediated acute lung injury and necrotic cell death. Nat Med. 2006;12(11):1286-93.

161. Bhandari $\mathrm{V}$, et al. Increased hyperoxia-induced lung injury in nitric oxide synthase 2 null mice is mediated via angiopoietin 2. Am J Respir Cell Mol Biol. 2012;46(5):668-76.

162. Olivier NB. Pulmonary edema. Vet Clin N Am Small Anim Pract. 1985;15(5):1011-30.

163. Bhatt AJ, et al. Disrupted pulmonary vasculature and decreased vascular endothelial growth factor, Flt-1, and TIE-2 in human infants dying with bronchopulmonary dysplasia. Am J Respir Crit Care Med. 2001;164(10 Pt 1):1971-80.

164. Rondelet B, et al. Signaling molecules in overcirculationinduced pulmonary hypertension in piglets: effects of sildenafil therapy. Circulation. 2004;110(15):2220-5.

165. Rondelet B, et al. Prevention of pulmonary vascular remodeling and of decreased BMPR-2 expression by losartan therapy in shunt-induced pulmonary hypertension. Am J Physiol Heart Circ Physiol. 2005;289(6):H2319-24.

166. Du L, et al. Signaling molecules in nonfamilial pulmonary hypertension. N Engl J Med. 2003;348(6):500-9.

167. Zhao YD, et al. Protective role of angiopoietin-1 in experimental pulmonary hypertension. Circ Res. 2003;92(9):984-91.

168. Miao H, et al. Novel angiogenesis strategy to ameliorate pulmonary hypertension. J Thorac Cardiovasc Surg. 2021;161(6):e417-e434.

169. Kugathasan L, et al. Role of angiopoietin- 1 in experimental and human pulmonary arterial hypertension. Chest. 2005;128(6 Suppl):633S-642S.

170. Dewachter L, et al. Angiopoietin/Tie2 pathway influences smooth muscle hyperplasia in idiopathic pulmonary hypertension. Am J Respir Crit Care Med. 2006;174(9):1025-33.

171. Richter MJ, et al. Circulating angiopoietin-1 is not a biomarker of disease severity or prognosis in pulmonary hypertension. PLoS ONE. 2016;11(11):e0165982.

172. Noda S, et al. Serum Tie2 levels: clinical association with microangiopathies in patients with systemic sclerosis. J Eur Acad Dermatol Venereol. 2011;25(12):1476-9.

173. Saleby J, et al. Angiogenic and inflammatory biomarkers in the differentiation of pulmonary hypertension. Scand Cardiovasc J. 2017;51(5):261-70.

174. Jonigk D, et al. Plexiform lesions in pulmonary arterial hypertension composition, architecture, and microenvironment. Am J Pathol. 2011;179(1):167-79.

175. Kugathasan L, et al. The angiopietin-1-Tie2 pathway prevents rather than promotes pulmonary arterial hypertension in transgenic mice. J Exp Med. 2009;206(10):2221-34.

176. Hiremath $\mathrm{J}$, et al. Exercise improvement and plasma biomarker changes with intravenous treprostinil therapy for pulmonary arterial hypertension: a placebo-controlled trial. J Heart Lung Transplant. 2010;29(2):137-49.
177. Kumpers $\mathrm{P}$, et al. Circulating angiopoietins in idiopathic pulmonary arterial hypertension. Eur Heart J. 2010;31(18):2291-300.

178. Hidalgo $M$, et al. First-in-human phase i study of single-agent vanucizumab, a first-in-class bispecific anti-angiopoietin-2/antiVEGF-A antibody, in adult patients with advanced solid tumors. Clin Cancer Res. 2018;24(7):1536-45.

179. Peplinski BS, et al. Associations of angiopoietins with heart failure incidence and severity. J Card Fail. 2021;27(7):786-95.

180. McDonald DM. Angiogenesis and remodeling of airway vasculature in chronic inflammation. Am J Respir Crit Care Med. 2001;164(10 Pt 2):S39-45.

181. Feistritzer C, et al. Expression and function of the angiopoietin receptor Tie-2 in human eosinophils. J Allergy Clin Immunol. 2004;114(5):1077-84.

182. Feltis BN, et al. Increased vascular endothelial growth factor and receptors: relationship to angiogenesis in asthma. Am J Respir Crit Care Med. 2006;173(11):1201-7.

183. Kanazawa H, Nomura S, Asai K. Roles of angiopoietin-1 and angiopoietin-2 on airway microvascular permeability in asthmatic patients. Chest. 2007;131(4):1035-41.

184. Kanazawa H, Tochino Y, Asai K. Angiopoietin-2 as a contributing factor of exercise-induced bronchoconstriction in asthmatic patients receiving inhaled corticosteroid therapy. J Allergy Clin Immunol. 2008;121(2):390-5.

185. Kanazawa $\mathrm{H}$, et al. Increased levels of angiopoietin-2 in induced sputum from smoking asthmatic patients. Clin Exp Allergy. 2009;39(9):1330-7.

186. Tseliou E, et al. Increased levels of angiopoietins 1 and 2 in sputum supernatant in severe refractory asthma. Allergy. 2012;67(3):396-402.

187. Moon KY, et al. Serum angiopoietin is associated with lung function in patients with asthma: a retrospective cohort study. BMC Pulm Med. 2014;14:143.

188. Koksal BT, et al. Evaluation of angiopoietin 1 and 2, vascular endothelial growth factor, and tumor necrosis factor alpha levels in asthmatic children. Allergy Asthma Proc. 2014;35(6):482-8.

189. Lee $\mathrm{PH}$, et al. Circulating angiopoietin- 1 and -2 in patients with stable and exacerbated asthma. Ann Allergy Asthma Immunol. 2016;116(4):339-43.

190. Makowska JS, et al. Angiopoietin-2 concentration in serum is associated with severe asthma phenotype. Allergy Asthma Clin Immunol. 2016;12:8.

191. Simoes DC, et al. Angiopoietin-1 protects against airway inflammation and hyperreactivity in asthma. Am J Respir Crit Care Med. 2008;177(12):1314-21.

192. Makinde TO, Agrawal DK. Increased expression of angiopoietins and Tie 2 in the lungs of chronic asthmatic mice. Am J Respir Cell Mol Biol. 2011;44(3):384-93.

193. Halim NSS, et al. Aerosolised mesenchymal stem cells expressing angiopoietin- 1 enhances airway repair. Stem Cell Rev Rep. 2019;15(1):112-25.

194. Gal Z, et al. Investigation of the possible role of Tie2 pathway and TEK gene in asthma and allergic conjunctivitis. Front Genet. 2020;11:128.

195. Fodor LE, et al. Variation in the TEK gene is not associated with asthma but with allergic conjunctivitis. Int J Immunogenet. 2018;45(3):102-8.

196. Naserghandi A, Allameh SF, Saffarpour R. All about COVID-19 in brief. New Microbes New Infect. 2020;35:100678.

197. Monteil V, et al. Inhibition of SARS-CoV-2 infections in engineered human tissues using clinical-grade soluble human ACE2. Cell. 2020;181(4):905-913.e7.

198. Chang L, Yan Y, Wang L. Coronavirus disease 2019: coronaviruses and blood safety. Transfus Med Rev. 2020;34(2):75-80.

199. Varga Z, et al. Endothelial cell infection and endotheliitis in COVID-19. Lancet. 2020;395(10234):1417-8. 
200. Smadja DM, et al. Angiopoietin-2 as a marker of endothelial activation is a good predictor factor for intensive care unit admission of COVID-19 patients. Angiogenesis. 2020;23(4):611-20.

201. Qanadli SD, Beigelman-Aubry C, Rotzinger DC. Vascular changes detected with thoracic CT in coronavirus disease (COVID-19) might be significant determinants for accurate diagnosis and optimal patient management. AJR Am J Roentgenol. 2020;215(1):W15.

202. Teuwen LA, et al. COVID-19: the vasculature unleashed. Nat Rev Immunol. 2020;20(7):389-91.

203. Bermejo-Martin JF, et al. Viral RNA load in plasma is associated with critical illness and a dysregulated host response in COVID19. Crit Care. 2020;24(1):691.

204. Villa E, et al. Dynamic angiopoietin-2 assessment predicts survival and chronic course in hospitalized patients with COVID-19. Blood Adv. 2021;5(3):662-73.

205. Sugiyama MG, et al. The Tie2-agonist vasculotide rescues mice from influenza virus infection. Sci Rep. 2015;5:11030.

206. Kumpers $\mathrm{P}$, et al. The synthetic tie 2 agonist peptide vasculotide protects against vascular leakage and reduces mortality in murine abdominal sepsis. Crit Care. 2011;15(5):R261.

207. David S, et al. Effects of a synthetic PEG-ylated Tie-2 agonist peptide on endotoxemic lung injury and mortality. Am J Physiol Lung Cell Mol Physiol. 2011;300(6):L851-62.
208. Van Slyke P, et al. Acceleration of diabetic wound healing by an angiopoietin peptide mimetic. Tissue Eng Part A. 2009;15(6):1269-80.

209. Tournaire R, et al. A short synthetic peptide inhibits signal transduction, migration and angiogenesis mediated by Tie 2 receptor. EMBO Rep. 2004;5(3):262-7.

210. Wu FT, et al. Vasculotide reduces endothelial permeability and tumor cell extravasation in the absence of binding to or agonistic activation of Tie2. EMBO Mol Med. 2015;7(6):770-87.

211. Vasomune. Vasomune announces initiation of the first-in-human clinical trial of a potential vascular normalization COVID-19 treatment. 2020. https://vasomune.com/vasomune-announcesinitiation-of-the-first-in-human-clinical-trial-of-a-potential-vascu lar-normalization-covid-19-treatment/.

212. ClinicalTrials.gov, N. A First-in-Human Study of AV-001 in Healthy Subjects. 2021. Clin Trial Ident: NCT04737486

213. Martin-Liberal J, et al. First-in-human, dose-escalation, phase 1 study of anti-angiopoietin-2 LY3127804 as monotherapy and in combination with ramucirumab in patients with advanced solid tumours. Br J Cancer. 2020;123(8):1235-43.

214. Patel S, Saxena B, Mehta P. Recent updates in the clinical trials of therapeutic monoclonal antibodies targeting cytokine storm for the management of COVID-19. Heliyon. 2021;7(2):e06158. 